X. Benveniste

Nagoya Math. J.

Vol. 97 (1985), 137-167

\title{
SUR LES VARIÉTÉS CANONIQUES DE DIMENSION 3 D'INDICE POSITIF
}

\author{
X. BENVENISTE
}

Dans tout ce qui suit, les varietés qui interviendront seront définies sur le corps des nombres complexes $C$. L'objet de cet article est de préciser le résultat suivant obtenu dans [B-2] et [K-1]:

THÉonìme 0 . Soit $X$ une variété projective normale de dimension 3, dont les singularités sont canoniques au sens de [R-1]. Soient e le p.p.c.m. des indices des points singuliers de $X$. Soit $R$ un diviseur de Cartier sur $X$, tel que le bidual de $\omega_{X}^{\otimes e}$ soit isomorphe à $\mathcal{O}_{X}(R)$. Supposons que $R$ soit numériquement positif et que $R^{3}>0$. Alors il existe un entier $n_{0}>0$ tel que pour tout $n \geqslant n_{0}$ le système linéaire $|n R|$ soit sans point base; en particulier l'anneau $\oplus_{n \geqslant 0} H^{0}\left(X, \mathcal{O}_{X}(n R)\right)$ est de type fini sur $C$.

par le résultat effectif suivant:

ThÉonk̀me 1. Avec les hypothèses du théorème 0 , pour tout entier $n \geqslant$ $34 e+3$, le système linéaire $|n R|$ est sans point base.

Si $e=1$, on peut être plus précis:

THÉORÈme 2. Avec les hypothèses du théorème 0 , supposons que $e=1$. Alors pour tout entier $m \geqslant 34$, le système linéaire $|m R|$ est sans point base et définit un morphisme birationnel sur son image.

Pour montrer ces deux théorèmes, nous aurons besoin du résultat suivant:

Proposition 1. Soit $X$ une variété normale et projective, dont les singularités sont canoniques au sens de [R-1]. Soient e le p.p.c.m. des indices des points singuliers de $X, R$ un diviseur de Cartier sur $X$, tels que le bidual de $\omega_{X}^{\otimes e}$ soit isomorphe à $\mathcal{O}_{X}(R)$. Soit $D$ un diviseur de Cartier sur $X$ tel que les diviseurs $D$ et $(e D-R)$ soient numériquement positifs et

Received January 23, 1984. 
que $(e D-R)^{3}>0$. Supposons $|4 D| \neq \emptyset$ et $|5 D| \neq \emptyset$ et soit $m$ un entier $\geqslant 17$. Si le système linéaire $|m D|$ n'est pas composé d'un pinceau, l'élément général de $|m D|$ est réduit, irréductible et n'a que des singularités isolées.

Nous consacrons le premier chapitre de cet article à la démonstration de la proposition. Ensuite nous intéressons aux surfaces $\boldsymbol{Q}$-Gorenstein de type général. Enfin nous démontrons les théorèmes 1 et 2 .

Désignons par $k$ l'un des deux corps $\boldsymbol{Q}$ ou $\boldsymbol{R}$. Pour toute variété $V$ lisse et projective, nous notons $N S_{k}(V)$ le $k$-espace vectoriel obtenu à partir de $N S(V)$ par extension des scalaires à $k$.

Etant donné une variété projective quelconque $X$, nous notons $\operatorname{Sing}(X)$ le lieu singulier de $X$.

Pour tout nombre réel $x$, nous notons $[x]$ et $\{x\}$ respectivement la partie entière de $X$ et le plus petit entier $\geqslant x$.

Qu'il nous soit permis ici de remercier A. Beauville pour ses précieux conseils lors de la rédaction de cet article.

\section{Quelques rappels et notations}

Rappelons le théorème suivant dû à Kawamata et Viehweg ([K] et [V]):

ThÉoRìme 0-1 (Kawamata, Viehweg). Soient $V$ une variété lisse et projective de dimension $d,\left(E_{i}\right)_{i \in I}$ une famille finie de diviseurs lisses $\grave{a}$ croisements normaux, $\left(a_{i}\right)_{i \in I}$ une famille de nombres rationnels de $[0,1[$, $D$ un élément de Pic $(V), L$ un élément de $N S_{Q}(V)$ numériquement positif vérifiant $L^{d}>0$, tels que l'on ait dans $N S_{Q}(V): D=L+\sum_{i \in I} a_{i} E_{i}$. Alors pour tout $p \leqslant d-1$, on $a: H^{p}\left(V, \mathcal{O}_{V}(-D)\right)=0$.

Donnons le résultat de [E] et [S]:

ThÉoRÈme 0-2 (Elkik, Shepherd-Barron). Soit $X$ une variété normale et projective de dimension 3 , n'ayant que des singularités canoniques au sens de [R-1]. Soient $Y$ un modèle lisse de $X, f: Y \rightarrow X$ un morphisme birationnel; alors on a $R^{p} f_{*} \mathcal{O}_{Y}=0$ pour $p>0$, et $f_{*} \mathcal{O}_{Y}=\mathcal{O}_{X}$.

Rappelons aussi le résultat de Reid [R-2]:

ThÉoRìme 0-3 (Reid). Soit $X$ une variété normale et projective de dimension 3 n'ayant que des singularités canoniques. Notons $\omega_{X}$ le faisceau dualisant de $X$. Alors il existe deux modèles birationnels $Y$ et $X^{\prime}$, deux morphismes birationnels $g: X^{\prime} \rightarrow X$ et $h: Y \rightarrow X^{\prime}$, une famille finie $\left(W_{i}\right)_{i \in I}$ de diviseurs lisses à croisements normaux sur $Y$, une famille $\left(\rho_{i}\right)_{i \in I}$ de 
nombres entiers $>0$, vérifiant les conditions suivantes:

(i) $Y$ est lisse et $X^{\prime} n^{\prime}$ a que des singularités terminales au sens de [R-2]. Notons $r$ le p.p.c.m. des indices des points singuliers de $X^{\prime}$.

(ii) Notons $\omega_{Y}$ et $\omega_{X^{\prime}}$ les faisceaux dualisants de $Y$ et $X^{\prime}$ respectivement, $\omega_{X}^{[r]}$ et $\omega_{X^{\prime}}^{[r]}$ les biduaux des faisceaux $\omega_{X}^{\otimes r}$ et $\omega_{X^{\prime}}^{\otimes r}$. Alors on $a$ :

$$
\omega_{X^{\prime}}^{[r]}=g^{*}\left(\omega_{X}^{[r]}\right), \text { et } \omega_{Y}^{\otimes r}=h^{*}\left(\omega_{X^{\prime}}^{[r]}\right)\left(\sum_{i \in I} \rho_{i} W_{i}\right) .
$$

(iii) Pour tout $i \in I, h\left(W_{i}\right)$ est un point singulier de $X^{\prime}$.

Le théorème 0 entraîne:

Proposition 0-1. Soit $X$ une variété de dimension 3 vérifiant les hypothèses $d u$ théorème 0. Posons $X_{c}=\operatorname{Proj}\left(\oplus_{n \geqslant 0} H^{0}\left(X, \mathcal{O}_{X}(n R)\right)\right)$. Alors $X_{c}$ est une variété normale de dimension 3 , n'ayant que des singularités canoniques au sens de [R-1]. Soit $\omega_{X_{c}}$ son faisceau dualisant; le bidual $\mathscr{L}$ du faisceau $\omega_{X_{c}}^{\otimes e}$ est inversible et ample. De plus il existe un morphisme birationnel $f: X \rightarrow X_{0}$ tel que $: f^{*}(\mathscr{L})=\mathcal{O}_{X}(R)$.

D'après la proposition $0-1$, quitte à considérer $X_{c}$ au lieu de $X$, il suffit de démontrer le théorème 1 dans le cas où $R$ est ample. Désormais nous allons considérer une variété normale $X$ de dimension 3, n'ayant que des singularités canoniques. Nous allons noter $r$ le p.p.c.m. des indices des points singuliers de $X$. Soit $\omega_{X}$ le faisceau dualisant de $X$. Soit $H$ un diviseur de Cartier ample sur $X$, tel que le bidual de $\omega_{X}^{\otimes r}$ soit isomorphe à $\mathcal{O}_{X}(H)$. Soient $X^{\prime}$ et $Y$ les modèles birationnels de $X$ définis dans le théorème $0-3$. Posons, avec les notations du théorème $0-3, f=$ $g \circ h$.

Proposition 0-2. On a les assertions suivantes:

(i) Pour tout entier $n<0$, et tout $p \in\{0,1,2\}$, on $a$ :

$$
H^{p}\left(X, \mathcal{O}_{X}(n H)\right)=H^{p}\left(Y, \mathcal{O}_{Y}\left(n f^{*}(H)\right)\right)=0 .
$$

(ii) Pour tout entier $n>0$, et tout $p \in\{1,2,3\}$, si $r \geqslant 2$, on $a$ :

$$
H^{p}\left(X, \mathcal{O}_{X}(n H)\right)=H^{p}\left(Y, \mathcal{O}_{Y}\left(n f^{*}(H)\right)\right)=0 .
$$

Démonstration. D'après la suite spectrale de Leray, et le théorème $0-2$, on a pour $p \geqslant 0$ et tout entier $n: H^{p}\left(X, \mathcal{O}_{X}(n H)\right)=H^{p}\left(Y, \mathcal{O}_{Y}\left(n f^{*}(H)\right)\right)$.

Si $n<0$, on d'après le théorème $0-1: H^{p}\left(Y, \mathcal{O}_{Y}\left(n f^{*}(H)\right)\right)=0$, pour $p<3$. Cela démontre l'assertion (i). 
Supposons $n>0$ et $r \geqslant 2$. Notons $K_{Y}$ le diviseur canonique de $Y$ et soient $\left(W_{i}\right)_{i \in I}$ et $\left(\rho_{i}\right)_{i \in I}$ les familles définies dans le théorème 0-3. La condition (ii) du théorème 0-3 montre que: $r K_{Y} \equiv f^{*}(H)+\sum_{i \in I} \rho_{i} W_{i}$. Posons $R \equiv f^{*}(H)$. Pour chaque $i \in I$, soit $a_{i}$ le plus petit entier $\geq \rho_{i} / r$; posons $c_{i}=a_{i}-\left(\rho_{i} / r\right)$ et $W=\sum_{i \in I} a_{i} W_{i}$. Dans $N S_{Q}(Y)$ on a:

$$
n R=K_{Y}+(n-(1 / r)) R+\sum_{i \in I} c_{i} W_{i}-W .
$$

Posons $L(n) \equiv n R-K_{Y}+W$; par définition on a: $n R \equiv K_{Y}+L(n)-W$. Dans $N S_{Q}(Y)$, on peut écrire: $L(n)=(n-(1 / r)) R+\sum_{i \in I} c_{i} W_{i}$. Remarquons que pour tout $i \in I, c_{i}$ est un nombre rationnel de [0,1[. Comme $(n-(1 / r))>0$ le théorème $0-1$ montre que:

$$
H^{p}\left(Y, \mathcal{O}_{Y}\left(K_{Y}+L(n)\right)\right)=0
$$

pour $p>0$. D'après la condition (iii) du théorème $0-3$, on a pour tout $i \in I:\left.R\right|_{W_{i}} \equiv 0$; comme $n R+W \equiv K_{Y}+L(n)$, on déduit: $\mathcal{O}_{W}\left(K_{Y}+L(n)\right)=$ $\mathcal{O}_{W}(W)$. Puisque les parties mobiles de $|n R|$ et $\left|n r K_{Y}\right|$ sont les mêmes, $W$ est contenu dans la partie fixe de $|n R+W|$. On a la suite exacte:

$$
0 \longrightarrow \mathcal{O}_{Y}(n R) \longrightarrow \mathcal{O}_{Y}\left(K_{Y}+L(n)\right) \longrightarrow \mathcal{O}_{W}(W) \longrightarrow 0 \text {. }
$$

D'après (1), pour tout entier $p>0$, l'homomorphisme:

$$
H^{p-1}\left(W, \mathcal{O}_{W}(W)\right) \longrightarrow H^{p}\left(Y, \mathcal{O}_{Y}(n R)\right)
$$

est un isomorphisme. D'après le théorème d'annulation de Serre sur les faisceaux amples, il existe un entier $n_{0}>0$ tel que pour tout $n \geqslant n_{0}$, on ait pour $p>0: H^{p}\left(X, \mathcal{O}_{X}(n H)\right)=0$. Par conséquence, on a:

$$
H^{p}\left(Y, \mathcal{O}_{Y}(n R)\right)=H^{p}\left(X, \mathcal{O}_{X}(n H)\right)=0
$$

pour $p>0$ et $n \geqslant n_{0}$. Cela implique: $H^{p-1}\left(W, \mathcal{O}_{W}(W)\right)=0$, pour $p>0$, et donc pour tout $n>0$, on a: $H^{p}\left(Y, \mathcal{O}_{Y}(n R)\right)=0$. Cela termine la démonstration de l'assertion (ii).

Proposition 0-3. Pour tout entier $n \geqslant 4$, on a les assertions suivantes:

(i) On $a: h^{0}\left(X, \mathcal{O}_{X}(n H)\right) \geqslant 7$.

(ii) Le systéme linéaire $|n H|$ n'est pas composé d'un pinceau.

Démonstration. Soit $n$ un entier $>0$. Reprenons les notations du théorème $0-3$. Posons $R \equiv f^{*}(H)$. D'après la proposition $0-2$, si $r \geqslant 2$, on a:

$$
h^{0}\left(X, \mathcal{O}_{X}(n H)\right)=\chi\left(Y, \mathcal{O}_{Y}(n R)\right)
$$


Si $r=1$, on a $\omega_{X} \cong \mathcal{O}_{X}(H)$. Pour $n \geqslant 2$, d'après la dualité de Serre, et l'assertion (i) de la proposition $0-2$, on a:

$$
h^{0}\left(X, \mathcal{O}_{X}(n H)\right)=\chi\left(Y, \mathcal{O}_{Y}(n R)\right) \text {. }
$$

Posons $Q(n)=\chi\left(Y, \mathcal{O}_{Y}(n R)\right)$. Appliquons le théorème de Riemann-Roch:

$$
Q(n)=n^{3}\left(R^{3} / 6\right)-n^{2}\left(\left(K_{Y} \cdot R^{2}\right) / 4\right)+n\left(R \cdot\left(K_{Y}^{2}+c_{2}(Y)\right) / 12\right)+\chi\left(\mathcal{O}_{Y}\right) .
$$

D'après l'assertion (ii) du théorème $0-3$, on a:

$$
r K_{Y} \equiv R+\sum_{i \in I} \rho_{i} W_{i} .
$$

D'après l'assertion (iii) du même théorème, on a: $\left.R\right|_{W_{i}} \equiv 0$, pour $i \in I$. Cela implique: $K_{Y} \cdot R^{2}=R^{3} / r$, et $K_{Y}^{2} \cdot R=R^{3} / r^{2}$. On déduit pour $n>0$ et $r \geqslant 2$ ou bien $n \geqslant 2$ et $r>0$ :

$$
Q(n)=n(2 n r-1)(n r-1)\left(H^{3} / 12 r^{2}\right)+n\left(c_{2}(Y) \cdot R / 12\right)+\chi\left(\mathcal{O}_{Y}\right)
$$

D'après l'assertion (i) de la proposition $0-2$, on a:

$$
h^{0}\left(Y, \mathcal{O}_{Y}\left(K_{Y}+R\right)\right)=\chi\left(\mathcal{O}_{Y}\left(K_{Y}+R\right)\right) .
$$

Posons $a=h^{0}\left(Y, \mathcal{O}_{Y}\left(K_{Y}+R\right)\right)$; en appliquant le théorème Riemann-Roch et les remarques précédentes, on obtient:

$$
a=(2 r+1)(r+1)\left(H^{3} / 12 r^{2}\right)+\left(c_{2}(Y) \cdot R / 12\right)-\chi\left(\mathcal{O}_{Y}\right) .
$$

Distinguons maintenant deux cas:

$1^{\text {er }}$ cas: supposons $r \geqslant 2$.

Comme a est entier, $r^{2}$ divise $H^{3}$. Puisque $Q(1) \geqslant 0$, on déduit:

$$
\chi\left(\mathcal{O}_{Y}\right) \geqslant-\left(c_{2}(Y) \cdot R / 12\right)-(2 r-1)(r-1)\left(H^{3} / 12 r^{2}\right) .
$$

On a aussi: $Q(1)+a \geqslant 0$, ce qui donne:

$$
\left(c_{2}(Y) \cdot R / 12\right) \geqslant-\left(2 r^{2}+1\right)\left(H^{3} / 12 r^{2}\right) .
$$

En reportant la formule (3) dans la formule (1), on obtient pour $n \geqslant 2$ :

$$
Q(n) \geqslant\left(n\left(2 r^{2}\left(n^{2}-1\right)-3 n r\right)+\left(2 r^{2}+1\right)-(2 r-1)(r-1)\right) \cdot\left(H^{3} / 12 r^{2}\right)
$$

ce qui implique:

$$
Q(n) \geqslant n(n-1)(2 r(n+1)-3)\left(\left(H^{3} / 12 r\right) .\right.
$$

On déduit pour $n \geqslant 4: Q(n) \geqslant 3 \cdot 4 \cdot(10 r-3)\left(H^{3} / 12 r\right)$. Mais $r^{2}$ divise $H^{3}$, par suite: $\left(H^{3} / 12 r\right) \geqslant r / 12$, ce qui implique: $Q(n) \geqslant(10 r-3) r \geqslant 7$. Montrons l'assertion (ii). 
Soit $n$ un entier $>0$. Supposons le système linéaire $|n H|$ composé d'un pinceau. D'après le théorème de Bertini, il existe un diviseur irréductible $F_{n}$ et un diviseur $Z_{n}$, un entier $f(n)>0$, tels que:

$$
\begin{gathered}
n H \sim f(n) F_{n}+Z_{n}, \\
F_{n} \cdot H^{2} \geqslant 1 .
\end{gathered}
$$

Multiplions (6) par $H^{2}$ et utilisons la formule (7); on obtient:

$$
f(n) \leqslant n H^{3} .
$$

D'après (6), on a: $Q(n) \leqslant f(n)+1$. En utilisant (8), on obtient:

$$
Q(n) \leqslant n H^{3}+1 \text {. }
$$

En appliquant la formule (5), on déduit:

$$
n\left(2 r\left(n^{2}-1\right)-3(n-1)-12 r\right)\left(H^{3} / 12 r\right) \leqslant 1 .
$$

Mais pour $n \geqslant 4$, on a: $n\left(2 r n^{2}-3 n-14 r+3\right) \geqslant 4(18 r-9)$. Comme on a: $H^{3} / 12 r \geqslant r / 12$, cela implique:

$$
n\left(2 r n^{2}-3 n-14 r+3\right)\left(H^{3} / 12 r\right) \geqslant(18 r-9) / 3 \geqslant 3 .
$$

On obtient une contradiction avec (10). Cela achéve la démonstration du cas $r \geqslant 2$.

$$
2^{\text {ème }} \text { cas: supposons } r=1 \text {. }
$$

Par le théorème 0-2, on a pour $p>0: R^{p} f_{*} \mathcal{O}_{Y}=0$ et $f_{*} \mathcal{O}_{Y}=\mathcal{O}_{Y}$. La suite spectrale de Leray donne: $\chi\left(\mathcal{O}_{Y}\right)=\chi\left(\mathcal{O}_{X}\right)$. Par dualité de Serre sur $X$, on a: $\chi\left(\mathcal{O}_{X}\right)=-\chi\left(\mathcal{O}_{X}(H)\right)$. A nouveau, la suite spectrale de Leray donne: $\chi\left(\mathcal{O}_{Y}\left(f^{*}(H)\right)\right)=\chi\left(\mathcal{O}_{X}(H)\right)$. Par suite on a: $\chi\left(\mathcal{O}_{Y}\left(f^{*}(H)\right)=-\chi\left(\mathcal{O}_{Y}\right)\right.$. Posons comme précédemment $R=f^{*}(H)$. Appliquons le théorème de RiemannRoch on obtient:

$$
-\chi\left(\mathcal{O}_{Y}\right)=\left(R^{3} / 6\right)-\left(K_{Y} \cdot R^{2} / 4\right)+\left(R \cdot\left(K_{Y}^{2}+c_{2}(Y)\right) / 12\right)+\chi\left(\mathcal{O}_{Y}\right) .
$$

En utilisant la condition (iii) du théorème $0-3$, on voit que:

$$
K_{Y} \cdot R^{2}=R^{3} \quad \text { et } K_{Y}^{2} \cdot R=R^{3} .
$$

On déduit:

$$
2 \chi\left(\mathcal{O}_{Y}\right)=-\left(c_{2}(Y) \cdot R / 12\right) .
$$

(Remarque: on aurait pu écrire aussi: $\chi\left(\mathcal{O}_{Y}\left(K_{Y}\right)\right)=-\chi\left(\mathcal{O}_{Y}\right)$. On aurait obtenu: $2 \chi\left(\mathcal{O}_{Y}\right)=-\left(c_{2}(Y) \cdot K_{Y} / 12\right)$, ce qui montre que: $c_{2}(Y) \cdot \sum_{i \in I} \rho_{i} W_{i}=0$ ) 
La formule (2) donne: $a=h^{0}\left(Y, \mathcal{O}_{Y}\left(K_{Y}+R\right)\right)=\left(H^{3} / 2\right)+\left(c_{2}(Y) \cdot R / 12\right)-\chi\left(\mathcal{O}_{Y}\right)$. En utilisant (10), on obtient:

$$
a=\left(H^{3} / 2\right)-3 \chi\left(\mathcal{O}_{Y}\right) .
$$

Par suite 2 divise $H^{3}$. Pour tout entier $n \geqslant 2$, on a d'après les formules (1) et (11):

$$
Q(n)=n(2 n-1)(n-1)\left(H^{3} / 12\right)-(2 n-1) \chi\left(\mathcal{O}_{Y}\right) .
$$

Distinguons deux cas:

1) Supposons $H^{3}=2$; alors $\chi\left(\mathcal{O}_{Y}\right) \leqslant 0$ par la formule (12). La formule (13) montre que pour $n \geqslant 3$, on a:

$$
Q(n) \geqslant n(2 n-1)(n-1) / 6 .
$$

d'où on déduit pour $n \geqslant 4: Q(n) \geqslant(4 \cdot 7 \cdot 3) / 6 \geqslant 14$.

2) Supposons $H^{3} \geqslant 4$. Alors la formule (11) montre que pour $n \geqslant 3$ on a:

$$
Q(n) \geqslant((2 n-1) / 6)((n(n-1) / 2)-1) H^{3} .
$$

Pour $n \geq 4$, on déduit: $Q(n) \geqslant(7 / 6) \cdot 5 \cdot 4=(35 / 3) \geqslant 7$.

Montrons l'assertion (ii). Supposons que $|n H|$ soit composé d'un pinceau. Alors on a la formule (9). Distinguons deux cas:

1) Supposons $H^{3}=2$. Alors $\chi\left(\mathcal{O}_{Y}\right) \leqslant 0$ et on a d'après la formule (14):

$$
(n / 3)((2 n-1)(n-1)-1) \leqslant 1 .
$$

Mais pour $n \geqslant 4$, on a: $(n / 3)((2 n-1)(n-1)-1) \geqslant(4 / 3)(7 \cdot 3-1) \geqslant 24$, ce qui contredit la formule précédente.

2) Supposons $H^{3} \geqslant 4$. Alors d'après la formule (15) on obtient:

$$
((2 n-1)(n-1)(n / 12)-((2 n-1) / 6)-n) H^{3} \leqslant 1 .
$$

Mais on a: $(2 n-1)(n-1)(n / 12)-((2 n-1) / 6)-n=\left(n\left(2 n^{2}-3 n-15\right)\right.$ $+2) / 12$. On déduit pour $n \geqslant 4:((2 n-1)(n-1)(n / 12)-(2 n-1) / 6)-n) H^{3}$ $\geqslant 4 \cdot(11 / 6) \geqslant 7$. Cela donne une contradiction avec l'inégalité précédente, et termine la démonstration de la proposition.

Remarque 0-1. Soient $S$ une surface lisse et projective, $A$ et $B$ deux diviseurs numériquement positifs. Alors on a: $(A+B)^{2} \geqslant A^{2}$.

Nous renvoyons le lecteur au lemme 3-2 de [B-3] pour la démonstration de ce résultat. 
Proposition 0-4. Soient $S$ une surface lisse et projective de type général, $R$ un diviseur numériquement positif vérifiant $R^{2}>0$. Notons $K$ le diviseur canonique de $S$. Alors on $a:|K+R| \neq \emptyset$.

Démonstration. D'après la classification des surfaces, on a $\chi\left(\mathcal{O}_{S}\right)>0$. De plus le théorème $0-1$ implique: $H^{p}\left(S, \mathcal{O}_{S}(-R)\right)=0$ pour $p \leqslant 1$. Par le théorème de Riemann-Roch, on a: $h^{0}\left(S, \mathcal{O}_{S}(K+S)\right)=\chi\left(\mathcal{O}_{S}\right)+(1 / 2)((K+R) \cdot R)$. Puisque $S$ est de type général, on a: $K \cdot R \geqslant 0$ et donc: $h^{0}\left(S, \mathcal{O}_{S}(K+R)\right)$ $>0$. Cela termine la démonstration de la proposition.

D'après les résultats de [Ha] sur les éclatements on a:

Remarque 0-2. Soient $X$ et $Y$ deux variétés lisses, $f: Y \rightarrow X$ un morphisme birationnel composé d'une suite finie d'éclatements de centres lisses, $\left(E_{i}\right)_{i \in I}$ la famille des diviseurs exceptionnels de $f$, et $L$ un diviseur ample sur $X$. Alors il existe un entier $a>0$, et une famille $\left(a_{i}\right)_{i \in I}$ d'entiers $>0$ tels que le diviseur $f^{*}(L)-\sum_{i \in I} a_{i} E_{i}$ soit ample sur $Y$.

D'après les résultats de [B-1] on a:

Proposition 0-5. Soit Y une variété lisse et projective de dimension 3. Supposons que soient donnés:

a) un diviseur $P$ numériquement positif sur $Y$ vérifiant $P^{3}>0$,

b) une famille $\left(E_{i}\right)_{i \in I}$ de diviseurs lisses à croisements normaux,

c) un élément $o \in I$, une famille $\left(m_{i}\right)_{i \neq 0}$ d'entiers positifs, une famille $\left(c_{i}\right)_{i \in I}$ de nombres rationnels de $[0,1[$,

d) un $Q$-diviseur ample $L$ dans $N S_{Q}(Y)$, et un élément $G$ de $\operatorname{Pic}(Y)$, tels que dans $N S_{Q}(Y)$ on ait: $G=L+\sum_{i \neq 0} c_{i} E_{i}$,

e) un entier $m>0$ tel que si $D \equiv m P+\sum_{i \neq 0} m_{i} E_{i}$, on ait:

$$
D \equiv K_{Y}+P+G+E_{0} \text {. }
$$

Alors $E_{0}$ 'est pas partie fixe du système linéaire $|D|$.

Nous renvoyons à la proposition 2-1 de [B-1] pour la démonstration de ce résultat.

Rappelons maintenant les notations suivantes dues à Fujita [F]: Etant donnés une variété lisse et projective $V$ et un diviseur effectif $D$ sur $V$, on note $B(D)$ le lieu fixe du système linéaire $|D|$. 


\section{La démonstration de la proposition 1}

1) Une première proposition technique

Désormais $X$ désigne une variété de dimension 3 normale et projective dont les singularités sont canoniques au sens de [R-1]. Soient e le p.p.c.m. des indices des points singuliers de $X, H$ et $D$ deux diviseurs de Cartier, tels que:

- le bidual de $\omega_{X}^{\otimes e}$ soit isomorphe à $\mathcal{O}_{X}(H)$,

- les diviseurs $(e D-H)$ et $D$ sont numériquement positifs, et on a $(e D-H)^{3}>0$.

Nous allons faire les hypothèses suivantes (nous verrons au paragraphe 3 que l'on peut toujours les réaliser):

Soient $Y$ un modèle birationnel lisse de $X, f: Y \rightarrow X$ un morphisme birationnel, $\left(E_{i}\right)_{i \in I}$ une famille finie de diviseurs lisses à croisements normaux, $\left(\rho_{i}\right)_{i \in I}$ une famille de nombres rationnels positifs, $R=f^{*}(H)$ et $P=f^{*}(D)$, et $m$ un entier $\geqslant 3$, vérifiant les conditions suivantes:

$\mathrm{H}_{1}$ ) Il existe une famille $\left(u_{i}\right)_{i \in I}$ d'entiers positifs non tous nuls et un diviseur $M$ tels que: $m P \equiv M+\sum_{i \in I} u_{i} E_{i}, M$ est la partie mobile de $|m P|$, $\sum_{i \in I} u_{i} E_{i}$ sa partie fixe; de plus le système linéaire $|M|$ n'a pas de point base.

$\mathrm{H}_{2}$ ) Soit $K_{Y}$ le diviseur canonique de $Y$. On a dans $N S_{Q}(Y)$ :

$$
K_{Y}=(R / e)+\sum_{i \in I} \rho_{i} E_{i}
$$

Soit $i \in I$ tel que $\rho_{i}>0$, alors on a: $\operatorname{dim}\left(f\left(E_{i}\right)\right) \leqslant 1$.

$\mathrm{H}_{3}$ ) Il existe une famille $\left(t_{i}\right)_{i \in I}$ de nombres rationnels de ]0, 1[, et un élément $\tau \in] 0,1\left[\cap \boldsymbol{Q}\right.$, tels que pour toute famille $\left(\tau_{i}\right)_{i \in I}$ de nombres rationnels de $[0, \tau]$, le $Q$-diviseur $(P-(R / e))-\sum_{i \in I}\left(t_{i}+\tau_{i}\right) E_{i}$ est ample.

Proposition 1-1. Sous les hypothèses $H_{1}, H_{2}$ et $H_{3}$, on a pour tout $i \in I$ :

$$
u_{i} /\left(\rho_{i}+1\right) \leqslant 1+(2 /(m-2)) .
$$

Démonstration. Nous allons raisonner par l'absurde et supposer qu'il existe $\alpha \in I$ tel que: $u_{\alpha} /\left(\rho_{\alpha}+1\right)>1+(2 /(m-2))$. Nous allons montrer en plusieurs pas qu'il existe:

- un élément $o \in I$,

- une famille $\left(m_{i}\right)_{i \neq 0}$ d'entiers positifs,

- une famille de nombres rationnels $\left(c_{i}\right)_{i \neq 0}$ de nombres rationnels de $[0,1[$,

- un $\boldsymbol{Q}$-diviseur ample $L$, 
tels que si $A \equiv m P+\sum_{i \neq 0} m_{i} E_{i}$ les conditions a), $\cdots$, e) de la proposition 0-5 soient satisfaites. De plus $E_{0}$ est partie fixe du système linéaire $|A|$. Cela entraînera une contradiction avec la proposition 0-5.

$1^{e r}$ pas. quelques calculs arithmétiques:

Soit $r$ un nombre rationnel $>0$. Posons:

$$
a(r)=\max _{i \in I}\left(u_{i}+r\left(t_{i}+\tau\right)\right) /\left(\rho_{i}+1\right) .
$$

Soit $E(r)$ l'ensemble formé des $i \in I$ tels que:

$$
a(r)=\left(u_{i}+r\left(t_{i}+\tau\right)\right) /\left(\rho_{i}+1\right) .
$$

En faisant tendre $r$ vers 0 , on voit qu'il existe un nombre rationnel $r>0$ tel que les conditions suivantes soient réalisées:

(i) pour tout $i \in E(r)$, on a $u_{i} \neq 0$,

(ii) $a(r)>u_{\alpha} /\left(\rho_{\alpha}+1\right)$,

(iii) $r / a(r)<1$.

Soit $o \in E(r)$; posons $s_{0}=t_{0}+\tau$ et $s_{i}=t_{i}$ pour $i \in I-\{o\}$. Il est clair que si l'on définit pour tout $i \in I, m_{i}$ par:

$$
m_{i}=-\left[\left(\left(u_{i}+r s_{i}\right) / a(r)\right)-\rho_{i}\right],
$$

on a $m_{0}=-1$ et pour tout $i \in I-\{0\}: 0 \leqslant m_{i} \leqslant\left\{\rho_{i}\right\}$. De plus posons pour tout $i \in I-\{0\}, c_{i}=m_{i}+\left(\left(u_{i}+r s_{i}\right) / a(r)\right)-\rho_{i}$. On a: $c_{i} \in[0,1[$.

$2^{\text {ème }}$ pas. le diviseur $A \equiv m P+\sum_{i \neq 0} m_{i} E_{i}$ vérifie les hypothèses de la proposition $0-5$.

Montrons premièrement que $A$ vérifie l'hypothèse e). Ecrivons dans $N S_{Q}(Y)$ :

$$
\begin{aligned}
A-K_{Y}-E_{0 .}= & (m-(m / a(r))-2) P+(P-(R / e))-(r / a(r)) \sum_{i \in I} s_{i} E_{i} \\
& +(M / a(r))+P+\sum_{i \neq 0}\left(m_{i}+\left(\left(u_{i}+r s_{i}\right) / a(r)\right)-\rho_{i}\right) E_{i} .
\end{aligned}
$$

Posons dans $N S_{Q}(Y)$ :

$$
L=(m-(m / a(r))-2) P+(P-(R / e))-(r / a(r)) \sum_{i \in I} s_{i} E_{i},
$$

$L$ est un $\boldsymbol{Q}$-diviseur ample. En effet par construction on a:

$$
m-(m / a(r))-2 \geqslant m-\left(m\left(\rho_{\alpha}+1\right) / u_{\alpha}\right)-2>0,
$$

et par le choix de $r$ (condition (iii) du $1^{\mathrm{er}}$ pas) on a: $r / a(r)<1$. Par l'hypothèse $H_{3}$, le $Q$-diviseur $(P-(R / e))-\sum_{i \in I} s_{i} E_{i}$ est ample et l'on peut écrire: 


$$
\begin{aligned}
L= & (m-(m / a(r))-2) P+(1-(r / a(r)))(P-R / e)) \\
& +(r / a(r))\left((P-(R / e))-\sum_{i \in I} s_{i} E_{i}\right) .
\end{aligned}
$$

Cela montre que $L$ est un $\boldsymbol{Q}$-diviseur ample. Posons maintenant:

$$
G \equiv A-K_{Y}-P-E_{0} \text {. }
$$

On a par définition dans $N S_{Q}(Y): G=L+\sum_{i \neq 0} c_{i} E_{i}$. De plus on a:

$$
A \equiv K_{Y}+G+P+E_{0} \text {. }
$$

Cela montre que l'hypothèse $e$ ) de la proposition 0-5 est vérifiée. Le lecteur vérifiera sans peine que les conditions restantes sont remplies. Le $2^{\text {ème }}$ pas est donc démontré.

$3^{\text {ème }}$ pas. $E_{0}$ est contenu dans la partie fixe du système linéaire $|A|$.

Montrons que les parties mobiles de $|m P|$ et de $|A|$ sont les mêmes. D'après l'hypothèse $H_{2}$, on a pour tout $i \in I$ tel que $\rho_{i}>0: \operatorname{dim}\left(f\left(E_{i}\right)\right) \leqslant 1$. Par construction, on a pour tout $i \in I-\{o\}: 0 \leqslant m_{i} \leqslant\left\{\rho_{i}\right\}$. Par suite, si $\rho_{i}=0$, on $a m_{i}=0$. De plus, puisque $f_{*}\left(\mathcal{O}_{Y}\right)=\mathcal{O}_{X}$, on a les isomorphismes suivants: $H^{0}\left(X, \mathcal{O}_{X}(m D)\right) \cong H^{0}\left(Y, \mathcal{O}_{Y}(A)\right)$, et $H^{0}\left(X, \mathcal{O}_{X}(m D)\right) \cong H^{0}\left(Y, \mathcal{O}_{Y}(m P)\right)$. Cela démontre le résultat annoncé.

Par le choix de $r$ (condition (i) du $1^{\text {er }}$ pas) on a $u_{0} \neq 0$. Cela démontre le $3^{\text {ème }}$ pas.

D'après les considérations du début, on a donc démontré la proposition.

2) Une deuxième proposition technique.

Dans la section précédente, nous avons considéré la variété $Y$ au dessus de $X$ vérifiant les conditions $H_{1}, H_{2}$ et $H_{3}$. Nous allons supposer l'entier $m \geqslant 12$ et dès à présent considérer la situation obtenue en remplaçant la condition $H_{1}$ par $H_{1}^{\prime}$ :

$\left.\mathrm{H}_{1}^{\prime}\right)$ Il existe une famille $\left(u_{i}\right)_{i \in I}$ d'entiers positifs non tous nuls, pour chaque $n \in\{4,5\}$ une famille $\left(b_{i}(n)\right)_{i \in I}$ d'entiers positifs et un diviseur $M(n)$ tels que:

(i) $m P \equiv M+\sum_{i \in I} u_{i} E_{i}, M$ est la partie mobile de $|m P|, \sum_{i \in I} u_{i} E_{i}$ sa partie fixe. De plus $|M|$ est sans point base.

(ii) $n P \equiv M(n)+\sum_{i \in I} b_{i}(n) E_{i}, M(n)$ est la partie mobile de $|n P|$, $\sum_{i \in I} b_{i}(n) E_{i}$ sa partie fixe. De plus $|M(n)|$ est sans point base.

LEMME 1-1. Soit $\alpha \in I$ tel que $u_{\alpha} /\left(\rho_{\alpha}+1\right) \geqslant 1$; si $\rho_{\alpha} \in\{0,1\}$, il existe $n \in\{4,5\}$ tel que $b_{\alpha}(n) \geqslant 1$ 
Démonstration. Puisque $m \geqslant 12$, il existe deux entiers positifs $p$ et $q$ tels que: $m=4 p+5 q$. Puisque les multiplicités des composantes fixes des multiples d'un même diviseur sont sous-additives: $u_{\alpha} \leqslant p b_{\alpha}(4)+q b_{\alpha}(5)$. Mais on a: $u_{\alpha} \geqslant\left(\rho_{\alpha}+1\right) \geqslant 1$; on déduit qu'il existe $n \in\{4,5\}$ tel que $b_{\alpha}(n)$ $\geqslant 1$.

Nous allons raffiner la proposition 1-1:

Proposition 1-2. Sous les hypothèses $H_{1}^{\prime}, H_{2}$, et $H_{3}$ pour tout $m \geqslant 17$ et tout $i \in I$ tel que $\rho_{i} \in\{0,1\}$, on $a: u_{i} /\left(\rho_{i}+1\right)<1$.

Démonstration. Nous allons raisonner par l'absurde et supposer qu'il existe $\alpha \in I$ tel que $\rho_{\alpha} \in\{0,1\}$ et: $u_{\alpha} /\left(\rho_{\alpha}+1\right) \geqslant 1$. Remarquons que si $u_{\alpha} /\left(\rho_{\alpha}+1\right)>1$, on a en fait: $u_{\alpha} /\left(\rho_{\alpha}+1\right) \geqslant 1+(1 / 2)$. Puisque $m \geqslant 17$, on a aussi: $1+(2 /(\mathrm{m}-2)) \leqslant 1+(2 / 15)<1+(1 / 2)$. Cela contredirait la proposition 1-1. On peut donc supposer: $u_{\alpha} /\left(\rho_{\alpha}+1\right)=1$. Nous allons procéder en plusieurs pas:

$1^{\text {er }}$ pas: Il existe un entier $n \in\{4,5\}$ et un nombre rationnel $t>0$ vérifiant les conditions suivantes: Posons $b(t)=\max \left\{\left(u_{i}+t b_{i}(n)\right) /\left(\rho_{i}+1\right)\right.$, $i \in I\}$, et notons $F(t)$ l'ensemble formé des $i \in I$ tels que:

$$
b(t)=\left(u_{i}+t b_{i}(n)\right) /\left(\rho_{i}+1\right) .
$$

Alors on a:

(i ) $m-((m+t n) / b(t))-2>0$,

(ii) $u_{i} \neq 0$ pour tout $i \in F(t)$.

Remarquons que pour avoir la condition (i), il suffit de trouver $t$ et $n$ tels que: $m-\left((m+t n)\left(\rho_{\alpha}+1\right) /\left(u_{\alpha}+t b_{\alpha}(n)\right)\right)-2>0$, ce qui équivaut à: $m+t n<(m-2)\left(1+t b_{\alpha}(n)\right) /\left(\rho_{\alpha}+1\right)$, ou encore:

$$
t\left(\left((m-2) b_{\alpha}(n) /\left(\rho_{\alpha}+1\right)\right)-n\right)>2 .
$$

La condition (ii) est impliquée par la condition: pour tout $i \in I$, on a $t b_{i}(n) /\left(\rho_{i}+1\right)<\left(u_{\alpha}+t b_{\alpha}(n)\right) /\left(\rho_{\alpha}+1\right) \leqslant b(t)$. Mais d'après la proposition 1-1, on a pour tout $i \in I: b_{i}(n) /\left(\rho_{i}+1\right) \leqslant 1+(2 /(n-2))$. La condition (ii) sera réalisée dès que: $t(1+(2 /(n-2)))<1+\left(t b_{\alpha}(n) /\left(\rho_{\alpha}+1\right)\right)$, c'est à dire:

$$
t\left(1-\left(b_{\alpha}(n) /\left(\rho_{\alpha}+1\right)\right)+(2 /(n-2))<1 .\right.
$$

Posons $c_{\alpha}(n)=b_{\alpha}(n) /\left(\rho_{\alpha}+1\right)$; les conditions (A) et (B) sont compatibles si la condition suivante est réalisée:

$$
1+(n / 2)+(2 /(n-2))<m c_{\alpha}(n) / 2 \text { et }(m-2) c_{\alpha}(n)-n>0 .
$$

La condition (C) ne dépend plus de $t$. Nous allons montrer que celle-ci 
est satisfaite pour un $n \in\{4,5\}$ dès que $m \geqslant 17$.

Choisissons à l'aide du lemme 1-1 un entier $n \in\{4,5\}$ tel que $b_{\alpha}(n)$ $\geqslant 1$. On peut donc supposer $c_{\alpha}(n) \geqslant 1 / 2$; remarquons que:

$$
\begin{aligned}
& ((m-2) / 2)-n \geqslant(15 / 2)-5=5 / 2>0, \\
& 1+(n / 2)+(2 /(n-2)) \leqslant 4+(1 / 6), \\
& c_{\alpha}(n) m / 2 \geqslant m / 4 \geqslant 4+(1 / 4) .
\end{aligned}
$$

Cela démontre le résultat.

$2^{\text {ème }}$ pas. quelques calculs arithmétiques.

Soient $t$ un nombre rationnel $>0$ et $n \in\{4,5\}$ tels que les conditions (i) et (ii) du $1^{\mathrm{er}}$ pas soient réalisées. Posons avec les notations du $1^{\mathrm{er}}$ pas:

$$
c=m-((m+t n) / b(t))-2 .
$$

D'après le $1^{\mathrm{er}}$ pas on $a: c>0$. Posons pour chaque $i \in I: d_{i}=u_{i}+t b_{i}(n)$. Pour tout nombre rationnel $r>0$ soit:

$$
a(r)=\max \left\{\left(d_{i}+r\left(t_{i}+\tau\right)\right) /\left(\rho_{i}+1\right), i \in I\right\} .
$$

Notons comme précédemment $E(r)$ l'ensemble formé des $\mathrm{i} \in I$ tels que:

$$
a(r)=\left(d_{i}+r\left(t_{i}+\tau\right)\right) /\left(\rho_{i}+1\right) .
$$

En faisant tendre $r$ vers 0 , il est clair qu'il existe un nombre rationnel $r>0$ tel que les conditions suivantes soient remplies:

(i ) $m-((m+t n)) / a(r))-2>c$,

(ii) $E(r) \subset F(t)$,

(iii) $r / a(r)<1$.

D'après l'assertion (ii) du $1^{\text {er }}$ pas, la condition $E(r) \subset F(t)$ entraîne que pour tout $i \in E(r)$, on $a u_{i} \neq 0$. Soit $o \in E(r)$; posons $s_{0}=t_{0}+\tau$ et pour tout $i \in I-\{o\}: s_{i}=t_{i}$. On définit une famille $\left(m_{i}\right)_{i \neq 0}$ d'entiers positifs en posant pour chaque $i \neq 0$ :

$$
m_{i}=-\left[\left(\left(d_{i}+r s_{i}\right) / a(r)\right)-\rho_{i}\right] .
$$

On a: $0 \leqslant m_{i} \leqslant\left\{\rho_{i}\right\}$; de plus: $\left(\left(d_{0}+r s_{0}\right) / a(r)\right)-\rho_{0}=1$.

$3^{\text {ème }}$ pas. Le diviseur $A \equiv m P+\sum_{i \neq 0} m_{i} E_{i}$ vérifie les conditions a), $\cdots$, e) de la proposition $0-5$.

Avec les notations des pas antérieurs, écrivons dans $N S_{Q}(Y)$ :

$$
\begin{gathered}
A-K_{Y}-E_{0}=(m-((m+t n) / a(r))-2) P+(P-(R / e))-(r(a / r)) \sum_{i \in I} s_{i} E_{i} \\
+P+((M+t M(n)) / a(r))+\sum_{i \neq 0}\left(\left(\left(d_{i}+r s_{i}\right) / a(r)\right)+m_{i}-\rho_{i}\right) E_{i} .
\end{gathered}
$$


Pour tout $i \in I-\{o\}$, posons: $c_{i}=\left(\left(d_{i}+r s_{i}\right) / a(r)\right)+m_{i}-\rho_{i}$. Alors d'après la définition des $m_{i}, c_{i}$ est un nombre rationnel de [0,1[ pour $i \neq 0$. D'après les conditions (i) et (iii) du $2^{\text {ème }}$ pas relatives au choix de $r$, on a: $m-((m+t n) / a(r))-2>0$. Si l'on pose dans $N S_{Q}(Y)$ :

$$
L=(m-((m+t n) / a(r))-2) P+(P-(R / e))-(r / a(r)) \sum_{i \in I} s_{i} E_{\imath},
$$

on a:

$$
\begin{aligned}
L= & (m-((m+t n) / a(r))-2) P+(1-(r / a(r))(P-(R / e)) \\
& +(r / a(r))\left(P-(R / e)-\sum_{i \in I} s_{i} E_{i}\right) .
\end{aligned}
$$

D'après l'hypothèse $H_{3}$, il est facile de voir que $L$ est un $\boldsymbol{Q}$-diviseur ample. Posons maintenant: $G \equiv A-K_{Y}-E_{0}-P$. On a dans $N S_{Q}(Y): G=L+$ $\sum_{i \neq 0} c_{i} E_{i}$. Par définition on a: $A \equiv K_{Y}+E_{0}+P+G$. Le lecteur vérifiera sans peine que les conditions a), $\cdots$, e) de la proposition $0-5$ sont satisfaites. Cela termine la démonstration du $3^{\text {ème }}$ pas.

$4^{\text {ème }}$ pas. $E_{0}$ est contenu dans la partie fixe du système linéaire $|A|$. Remarquons que par le choix de $r$ effectué au $2^{\text {ème }}$ pas (condition (ii)), on a $u_{0} \neq 0$. Pour avoir le résultat, il suffit de reprendre la démonstration du $3^{\text {ème }}$ pas de la proposition 1-1; nous y renvoyons donc le lecteur.

Le fait que $E_{0}$ soit composante fixe du système linéaire $|A|$ contredit la proposition 0-5, et démontre la proposition 1-2.

3) Quelques constructions préparatoires

Soient $X, H$ et $D$ vérifiant les hypothèses de la proposition 1 .

Proposition 1-3. Soit $m$ un entier $\geqslant 12$. Il existe un modèle birationnel lisse $Y$ de $X$, un morphisme $f: Y \rightarrow X$ tels que:

(i) les hypothèses $H_{1}^{\prime}, H_{2}$ et $H_{3} d u$ deuxième paragraphe de ce chapitre soient satisfaites.

(ii) Pour toute composante irréductible $C$ de dimension 1 de $B(m D)$, avec les notations $d u 2^{\text {ème }}$ paragraphe de ce chapitre, il existe $i \in I$ vérifiant:

a) $f\left(E_{i}\right)=C$,

b) on a $\rho_{i}=0$ si $C \subset \operatorname{sing}(X)$,

c) on $a \rho_{i}=1$ si $C \not \subset \operatorname{Sing}(X)$.

Démonstration. Montrons l'assertion (i). En fait, nous allons seulement démontrer que les hypothèses $H_{1}, H_{2}$ et $H_{3}$ du $1^{\text {er }}$ paragraphe de ce chapitre peuvent être satisfaites, en laissant au lecteur le soin d'adapter la démonstration pour avoir $H_{1}^{\prime}$. Soient $m \geqslant 12, N$ la partie mobile de 
$|m D|,\left(W_{i}\right)_{i \in J_{1}}$ la famille finie des composantes irréductibles de dimension 2 de la partie fixe de $|m D|,\left(a_{i}\right)_{i \in J_{1}}$ une famille d'entiers positifs, tels que:

$$
m D \equiv N+\sum_{i \in J_{1}} a_{i} W_{i} .
$$

Soient $L$ un diviseur ample, $\left(W_{i}\right)_{i \in J_{2}}$ une famille finie de diviseurs irréductibles, $\left(h_{i}\right)_{i \in J_{2}}$ une famille d'entiers positifs, et $m_{0}$ un entier $>0$ tels que: $m_{0}(e D-H) \equiv L+\sum_{i \in J_{2}} h_{i} W_{i}$.

D'après le théorème $0-3$, soient $Z$ et $X^{\prime}$ deux modèles birationnels de $X, s: X^{\prime} \rightarrow X$ et $t: Z \rightarrow X^{\prime}$ deux morphismes birationnels, $\left(V_{f}\right)_{j \in J}$ une famille finie de diviseurs lisses à croisements normaux sur $Z,\left(p_{j}\right)_{j \in J}$ une famille d'entiers positifs, tels que:

1) $Z$ est lisse et $X^{\prime}$ n'a que des singularités terminales au sens de [R-2].

2) Notons $\omega_{Z}$ et $\omega_{X^{\prime}}$ les faisceaux dualisants de $Y$ et $X^{\prime}$ respectivement. Alors on a: $\omega_{X^{\prime}}^{[e]}=s^{*}\left(\omega_{X}^{[[]]}\right)$et $\omega_{Z}^{\otimes e}=t^{*}\left(\omega_{X^{\prime}}^{[e]}\right)\left(\sum_{j \in J} p_{j} V_{j}\right)$.

3) Pour tout $j \in J, t\left(V_{j}\right)$ est un point singulier de $X^{\prime}$.

Soient $n$ un entier $>0$ et $i: X \rightarrow \boldsymbol{P}^{n}$ le plongement de $X$ dans $\boldsymbol{P}^{n}$ défini par un multiple convenable de $L$. D'après Hironaka ([Hi]), il existe un modèle birationnel lisse $\hat{\boldsymbol{P}}^{n}$ de $\boldsymbol{P}^{n}$, un morphisme birationnel $\Phi: \hat{\boldsymbol{P}}^{n} \rightarrow \boldsymbol{P}^{n}$ composé d'une suite finie d'éclatements de centres lisses de dimension $\leqslant 2$, vérifiant les conditions suivantes: Le transformé strict $X_{0}$ de $X$ par $\Phi$ est lisse. Notons $f_{0}=\left.\Phi\right|_{X_{0}}$. Soient $\left(E_{i}^{0}\right)_{i \in I_{0}}$ la famille des diviseurs obtenue en prenant les composantes irréductibles des traces sur $X_{0}$ des diviseurs exceptionnels de $\Phi$. Alors pour tout $i \in I_{0}$, on a: $f_{0}\left(E_{i}^{0}\right) \subset \operatorname{Sing}(X)$.

Puisque $X_{0}$ est lisse, il existe un modèle birationnel lisse $X_{1}$ de $X_{0}$, un morphisme birationnel $f_{1}: X_{1} \rightarrow X_{0}$ composé d'une suite finie d'éclatements de centres lisses de dimension $\leqslant 1$, tels que la partie mobile de $\left|\left(f_{0} \circ f_{1}\right) *(m D)\right|$ soit sans point base. Posons $g=s \circ t$.

Soient $Y$ un modèle birationnel lisse de $X_{1}, f_{2}: Y \rightarrow X_{1}$ et $h: Y \rightarrow Z$ deux morphismes birationnels vérifiant les conditions suivantes:

$-f_{2}$ est composé d'une suite finie d'éclatements de centres lisses de dimension $\leqslant 1$.

- Posons $f=f_{0} \circ f_{1} \circ f_{2}$; soient $\left(E_{i}\right)_{i \in I}$ la famille formée des diviseurs exceptionnels de $f$ et des transformés stricts des $W_{j}$ pour $j \in J_{1} \cup J_{2}$ et des $E_{i}^{0}$ pour $i \in I_{0}$. Alors $\left(E_{i}\right)_{i \in I}$ est une famille de diviseurs lisses à croisements normaux.

-On a: $f=g \circ h$. 
Puisque la partie mobile de $\left|\left(f_{0} \circ f_{1}\right) *(m D)\right|$ est sans point base, il en est de même de celle de $\left|f^{*}(m D)\right|$. Soient $M$ la partie mobile de $\left|f^{*}(m D)\right|$, $\left(u_{i}\right)_{i \in I}$ une famille d'entiers non tous nuls, tels que:

$$
f^{*}(m D) \equiv M+\sum_{i \in I} u_{i} E_{i}
$$

L'hypothèse $H_{1}$ est donc réalisée.

Soit $K_{Y}$ le diviseur canonique de $Y$. Posons $R=f^{*}(H)$ et $P=f^{*}(D)$. D'après [R-1], puisque $f: Y \rightarrow X$ est une désingularisation de $X$, il existe une famille $\left(\rho_{i}\right)_{i \in I}$ de nombres rationnels positifs telle que dans $N S_{Q}(Y)$, on ait:

$$
K_{Y}=(R / e)+\sum_{i \in I} \rho_{i} E_{i}
$$

et pour tout $i \in I$ tel que $\rho_{i}>0$, on a: $\operatorname{dim}\left(f\left(E_{i}\right)\right) \leqslant 1$. Cela démontre $H_{2}$.

Soit $\left(d_{i}\right)_{i \in I}$ une famille d'entiers positifs telle que:

$$
m_{v}(e D-H) \equiv f^{*}(L)+\sum_{i \in I} d_{i} E_{i}
$$

Soit $I^{\prime}$ l'ensemble formé des $i \in I$, tels que $E_{i}$ soit le transformé strict d'un diviseur $E_{j}^{0}$ pour $j \in I_{0}$ ou bien d'un diviseur exceptionnel de $f_{1} \circ f_{2}$. Alors d'après la remarque 0-2 (le plongement de $X$ dans $\boldsymbol{P}^{n}$ est défini à partir d'un multiple convenable de $L$ ), il existe un entier $b^{\prime}>0$ et une famille $\left(b_{i}\right)_{i \in I^{\prime}}$ d'entiers $>0$, tels que le diviseur $b^{\prime} f^{*}(L)-\sum_{i \in I^{\prime}} b_{i} E_{i}$ soit ample. D'après les résultats de Serre sur les faisceaux amples (cf. par exemple [Ha]), il existe un entier $b>0$, et une famille $\left(t_{i}^{\prime}\right)_{i \in I}$ d'entiers $>0$, tels que pour toute famille $\left(e_{i}\right)_{i \in I} \in\{0,1\}^{I}$, le diviseur $b f^{*}(L)-\sum_{i \in I}\left(t_{i}^{\prime}+e_{i}\right) E_{i}$ soit ample. Soient $q$ un entier $>0$, et $\left(e_{i}\right)_{i \in I}$ une famille d'entiers de $\{0,1\}$; on peut écrire:

$$
\begin{aligned}
\left(q+m_{0} b\right)(e P-R) \equiv & q(e P-R)+\left(b f^{*}(L)-\sum_{i \in I}\left(t_{i}^{\prime}+e_{i}\right) E_{i}\right) \\
& +\sum_{i \in I}\left(b d_{i}+t_{i}^{\prime}+e_{i}\right) E_{i} .
\end{aligned}
$$

Dans $N S_{Q}(Y)$, on a:

$$
\begin{aligned}
(P- & (R / e))-\sum_{i \in I}\left(\left(b d_{i}+t_{i}^{\prime}+e_{i}\right) / e\left(q+b m_{0}\right)\right) E_{i} \\
& =\left(q(e P-R)+\left(b f^{*}(L)-\sum_{i \in I}\left(t_{i}^{\prime}+e_{i}\right) E_{i}\right)\right) / e\left(q+b m_{0}\right) .
\end{aligned}
$$

Par suite si $q$ est assez grand, et si l'on pose pour tout $i \in I$ :

$$
t_{i}=\left(b d_{i}+t_{i}^{\prime}\right) / e\left(q+b m_{0}\right) \quad \text { et } \quad \tau=\left(1 / e\left(q+b m_{0}\right)\right)
$$


on obtient:

- pour tout $i \in I$, on a: $t_{i} \in[0,1[$.

- pour toute famille $\left(\tau_{i}\right)_{i \in I}$ de nombres rationnels de $[0, \tau]$ le $\boldsymbol{Q}$-diviseur $(P-(R / e))-\sum_{i \in I}\left(t_{i}+\tau_{i}\right) E_{i}$ est ample.

L'hypothèse $H_{3}$ est donc réalisée.

Montrons l'assertion (ii). Soit $C$ une composante irréductible de dimension 1 de $B(m b)$. Distinguons deux cas:

Supposons $C \subset \operatorname{Sing}(X)$. Alors d'après [R-2], il existe un diviseur irréductible sur $X^{\prime}$ tel que $s(W)=C$. Puisque $C \subset B(m D), W$ est contenu dans $B\left(s^{*}(m D)\right)$. Soit $W^{\prime}$ le transformé strict de $W$ par $t$. Alors $W^{\prime}$ est contenu dans $B\left(g^{*}(m D)\right)$, et $W^{\prime}$ est distinct de tous les $V_{j}$ pour $j \in J$. Comme $C \subset B(m D)$, il existe $i \in I$ tel que le transformé strict de $W^{\prime}$ par $h$ soit $E_{i}$. Puisque $h: Y \rightarrow Z$ est un isomorphisme en colimension 1 , on a $\rho_{i}=0$ d'après ce que l'on vient de voir.

Supposons $C \not \subset \operatorname{Sing}(X)$. Puisque $f_{0}: X_{0} \rightarrow X$ est une désingularisation de $X$ telle que pour tout $i \in I_{0}$, on ait: $f_{0}\left(E_{i}^{0}\right) \subset \operatorname{Sing}(X), C$ possède un transformé strict $C^{\prime}$ par $f_{0}$. La courbe $C^{\prime}$ est aussi une composante irréductible de dimension 1 de $B\left(f_{0}^{*}(m D)\right)$. Puisque la partie mobile de $\left|\left(f_{0} \circ f_{1}\right)^{*}(m D)\right|$ est sans point base, il existe un diviseur exceptionnel $W$ de $f_{1}$ correspondant à l'éclatement de la courbe $C^{\prime}$. Il existe donc $i \in I$, tel que $E_{i}$ soit le transformé strict de $W$ par $f_{2}$, et on a $\rho_{i}=1$.

Cela termine la démonstration de la proposition.

\section{4) La démonstration de la proposition 1}

Soit $m$ un entier $\geqslant 17$ tel que $|m D|$ ne soit pas composé d'un pinceau. D'après le théorème de Bertini, on peut supposer $B(m D) \neq \emptyset$, car sinon il n'y a rien à démontrer.

D'après la proposition 1-3, il existe un modèle birationnel lisse $Y$ de $X$, un morphisme birationnel $f: Y \rightarrow X$ vérifiant les conditions $H_{1}^{\prime}, H_{2}$, et $H_{3}$ du $2^{\text {ème }}$ paragraphe de ce chapitre. En reprenant les notations de ce paragraphe distinguons plusieurs cas:

$1^{e r}$ cas. Supposons que $B(m D)$ ait une composante irréductible de dimension 2. Soit $E_{i}$ le transformé strict de celle-ci par $f$. Alors on a $\rho_{i}=0$ et $u_{i} \geqslant 1$. Par suite $u_{i} /\left(\rho_{i}+1\right) \geqslant 1$, ce qui contredit la proposition $1-2$.

$2^{\text {ème }}$ cas. D'après le premier cas, on peut supposer que $\operatorname{dim}(B(m D))$ 
$\leqslant 1$. Soit $C$ une composante irréductible de dimension 1 de $B(m D)$. Distinguons les deux possibilités suivantes:

a) Supposons $C$ contenue dans Sing $(X)$. D'après la proposition 1-3, il existe $i \in I$ tel que $f\left(E_{i}\right)=C$ et $\rho_{i}=0$. Par hypothèse, on a: $u_{i} \geqslant 1$, et donc: $u_{i} /\left(\rho_{i}+1\right) \geqslant 1$. Cela contredit la proposition 1-2.

b) Supposons $C$ non contenue dans Sing $(X)$. Supposons que l'élément général de $|m D|$ passe par $C$ avec multiplicité $\geqslant 2$. D'après la proposition $1-3$, il existe $i \in I$ tel que $f\left(E_{i}\right)=C$ et $\rho_{i}=1$. Comme on a: $u_{i} \geqslant 2$, on déduit: $u_{i} /\left(\rho_{i}+1\right) \geqslant 1$, ce qui contredit la proposition 1-2.

Pour avoir la proposition 1, il suffit maintenant d'utiliser le théorème de Bertini.

\section{Un résultat sur les surfaces $Q$-Gorenstein}

Definition 2-1. Soit $S$ une surface normale et projective. Nous dirons que $S$ est $\boldsymbol{Q}$-Gorenstein, si elle vérifie les conditions suivantes: $S$ est de Cohen-Macaulay; notons $\omega_{S}$ son faisceau dualisant. Alors il existe un entier $r>0$, tel que le bidual du faisceau $\omega_{S}^{\otimes r}$ soit inversible.

Definition 2-2. Soit $S$ une surface normale projective $\boldsymbol{Q}$-Gorenstein. Nous dirons que $S$ est de type général si un modèle lisse de $S$ est une surface de type général.

Proposition 2-0. Soient $S$ une surface normale $Q$-Gorenstein de type général, $\omega_{S}$ son faisceau dualisant, $n$ un entier $\geqslant 2$, a et $r$ deux entiers $>0, D$ et $U$ deux diviseurs de Cartier sur $S$, vérifiant les conditions suivantes:

a) Le bidual de $\omega_{S}^{\otimes r}$ est isomorphe à $\mathcal{O}_{S}(U)$.

b) Pour toute courbe $C$ sur $S$, on a $\operatorname{deg}_{C}\left(\mathcal{O}_{S}(D)\right) \geqslant 0$ et $\operatorname{deg}_{C}\left(\mathcal{O}_{S}(a D-U)\right.$ ) $\geqslant 0$.

c) $D^{2}>0$ et on $a h^{0}\left(S, \mathcal{O}_{S}(n D)\right) \geqslant 4$.

Alors le faisceau $\mathcal{O}_{S}(m D)$ est engendré par ses sections globales dans les deux cas suivants:

(i) $r \geqslant 2$ et $m \geqslant n+a+r-1$,

(ii) $r=1$ et $m \geqslant n+r+a$.

Le but de ce chapitre est de démontrer cette proposition en trois étapes.

1) Un résultat élémentaire sur les surfaces de type général

Soient $S$ une surface lisse de type général, $S_{0}$ son modèle minimal et 
$f: S \rightarrow S_{0}$ le morphisme birationnel correspondant. Notons $K$ le diviseur canonique de $S, K_{0}$ l'image inverse de celui de $S_{0}$ par $f$. Par définition, il existe une famille finie de diviseurs effectifs $\left(L_{i}\right)_{i \in I}$, une famille $\left(C_{p}\right)_{p \in I}$ de courbes rationnelles lisses, une famille de sous-ensembles $\left(I_{p}\right)_{p \in I}$, un ordre partiel sur $I$, tels que les conditions suivantes soient réalisées:

a) pour tout $i \in I$, on a: $L_{i}^{2}=-1, K_{0} \cdot L_{i}=0$,

b) pour tout $i, j \in I, i \neq j$, on a $L_{i} \cdot L_{j}=0$,

c) pour tout $p \in I$, et tout $i \in I_{p}$, on a $p<i$, et $L_{p}=C_{p}+\sum_{i \in I_{p}} L_{i}$,

d) on a: $K \equiv K_{0}+\sum_{i \in I} L_{i}$.

Proposition 2-1. Soit $R$ un diviseur numériquement positif non nul sur $S$, soit $J$ l'ensemble formé des $i \in I$ tels que $R \cdot L_{i} \geqslant 1$. Le diviseur $R+\sum_{i \in J} L_{i}$ est numériquement positif.

Démonstration. Pour tout $i \notin J$, on a $R \cdot L_{i}=0$. Remarquons que si $p$ et $q$ sont dans $I$, on a $L_{p} \cdot C_{q} \geqslant-1$, et on a égalité si et seulement si $p=q$. En effet par la condition c), on a:

$$
C_{q}=L_{q}-\sum_{i \in I_{q}} L_{i}
$$

Si $p \neq q$, on obtient: $L_{p} \cdot\left(L_{q}-\sum_{i \in I_{q}} L_{i}\right)=-\left(\sum_{i \in I_{q}} L_{i}\right) \cdot L_{p} \geqslant 0$.

Si $p=q$, on obtient: $L_{p} \cdot\left(L_{p}-\sum_{i \in I_{p}} L_{i}\right)=-1$, ce qui montre le résultat. Soit $p \in I$ tel que: $\left(\sum_{j \in J} L_{j}\right) \cdot C_{p}<0$. Alors $p \in J$, et on a:

$$
\left(\sum_{j \in J} L_{j}\right) \cdot C_{p}=L_{p} \cdot C_{p}+\left(\sum_{j \neq p} L_{j}\right) \cdot C_{p} \text {. }
$$

D'après ce qui précéde, $L_{p} \cdot C_{p}=-1$ et $L_{j} \cdot C_{p} \geqslant 0$ pour $j \neq p$, par suite pour tout $j \in J-\{p\}$, on a $L_{j} \cdot C_{p}=0$ et $\left(\sum_{j \in J} L_{j}\right) \cdot C_{p}=-1$. La relation c) montre que $I_{p} \cap J=\emptyset$. On déduit pour tout $i \in I_{p}: R \cdot L_{i}=0$. Pour tout $p \in J$, on a $R \cdot L_{p} \geqslant 1$, et puisque $C_{p}=L_{p}-\sum_{i \in I_{p}} L_{i}$, on obtient: $R \cdot C_{p} \geqslant 1$. Montrons que pour tout $p \in I$, on a: $\left(R+\sum_{j \in J} L_{j}\right) \cdot C_{p} \geqslant 0$. Distinguons deux cas:

1) supposons $p \in J$ :

D'après ce qui précéde, on a: $\left(\sum_{j \in J} L_{j}\right) \cdot C_{p} \geqslant-1$. Comme $R \cdot C_{p} \geqslant 1$, on a: $\left(R+\sum_{j \in J} L_{j}\right) \cdot C_{p} \geqslant 0$.

2) supposons $p \notin J$ :

Alors on a: $\left(\sum_{j \in J} L_{j}\right) \cdot C_{p} \geqslant 0$, et donc puisque $R$ est numériquement positif, on a le résultat. Cela achéve de montrer la proposition.

2) Une proposition technique sur les surfaces.

Lemme 2-1. Soient $S$ une surface lisse et projective, $R$ un diviseur numériquement positif verifiant $R^{2}>0$. Alors: 
(i ) Le nombre de courbes irréductibles deux à deux distinctes $C$ telles que $R \cdot C=0$ est fini.

(ii) Le nombre de diviseurs effectifs $E$ distincts deux à deux tels que $R \cdot E=0$ et $E^{2}=-1$, est fini.

Nous renvoyons au lemme 1-2 de [B-1] pour la démonstration de ce résultat.

Proposition 2-2. Soient $S$ une surface lisse et projective, $R$ et $P$ deux diviseurs numériquement positifs, $K$ le diviseur canonique de $S, n$ un entier $\geqslant 2$, tels que l'on ait:

a) $P^{2}>0$ et $R^{2}>0$

b) $h^{0}\left(S, \mathcal{O}_{S}(n R)\right) \geqslant 4$.

Supposons $|K+P+n R| \neq \emptyset$. On a les assertions suivantes:

(i) Soit $x \in B(K+P+n R)$. Alors il existe un diviseur effectif $E$ passant par $\{x\}$ vérifiant $P \cdot E=R \cdot E=0$ et $E^{2}=-1$.

(ii) Soit $C$ une courbe irréductible contenue dans $B(K+P+n R)$. Alors $C$ est une courbe rationnelle lisse vérifiant $R \cdot C=P \cdot C=0$.

Démonstration. Soit $x$ un point fermé de $\mathrm{S}$. Soient $S^{\prime}$ un modèle birationnel de $S, f: S^{\prime} \rightarrow S$ l'éclatement du point $x$, et $L$ le diviseur exceptionnel correspondant. Notons $K^{\prime}$ le diviseur canonique de $S^{\prime}$. Supposons que $x \in B(K+P+n R)$. D'après l'hypothèse $\mathrm{b})$, il existe une famille $\left(C_{i}\right)_{i \in I}$ de courbes irréductibles, et une famille $\left(a_{i}\right)_{i \in I}$ d'entiers positifs, tels que l'on ait:

$$
\sum_{i \in I} a_{i} C_{i} \in\left|f^{*}(n R)-2 L\right|
$$

$1^{\text {er }}$ pas. la 0-connexité de $n f^{*}(R)-2 L$.

Soit $D \in\left|f^{*}(n R)-2 L\right|$. Alors montrons que $D$ est 0 -connexe au sens de [Ra]. Soient $D_{1}$ et $D_{2}$ deux diviseurs effectifs non nuls sur $S^{\prime}, G_{1}$ et $G_{2}$ deux diviseurs effectifs sur $S, n_{i}$ des entiers pour $i \in\{1,2\}$, tels que l'on ait:

$-D_{i}=f^{*}\left(G_{i}\right)-n_{i} L$ pour $i \in\{1,2\}$,

$-G_{1}+G_{2} \sim n R$, et $n_{1}+n_{2}=2$.

Ecrivons dans $N S_{Q}(S)$, pour $i \in\{1,2\}: G_{i}=\left(R \cdot G_{i} / R^{2}\right) R+(-1)^{i} \xi$ avec $R \cdot \xi$ $=0$. On a:

$$
R \cdot G_{1}+G \cdot R_{2}=n R^{2}
$$

De plus on a: 


$$
D_{1} \cdot D_{2}=G_{1} \cdot G_{2}-n_{1} n_{2}=\left(\left(R \cdot G_{1}\right)\left(R \cdot G_{2}\right) / R^{2}\right)-\xi^{2}-n_{1} n_{2} .
$$

D'après le théorème de l'indice sur $S$, on obtient $\xi^{2} \leqslant 0$. Distinguons deux cas:

$1^{\text {er }}$ cas. supposons $R \cdot G_{1}>0$ et $R \cdot G_{2}>0$.

On a d'après la formule (1): $\left(R \cdot G_{1}\right)\left(R \cdot G_{2}\right) / R^{2} \geqslant\left(n R^{2}-1\right) / R^{2} \geqslant n-$ $\left(1 / R^{2}\right) \geqslant 2-1=1$. D'après la formule (2), on déduit: $D_{1} \cdot D_{2} \geqslant 0$.

$2^{\text {ème }}$ cas. supposons $R \cdot G_{1}=0$.

Alors on a: $\left(G_{1}+G_{2}\right) \cdot G_{1}=G_{1} \cdot G_{2}+G_{1}^{2}=0$. Puisque $R^{2}>0$, le théorème de l'indice sur $S$ implique: $G_{1}^{2} \leqslant-1$, ce qui montre que: $G_{1} \cdot G_{2} \geqslant 1$. En utilisant la formule (2), on obtient: $D_{1} \cdot D_{2} \geqslant 1-1=0$.

$2^{\text {ème }}$ pas. On a l'une des assertions suivantes:

(i) Il existe un entier $m>0$, une famille $\left(D_{p}\right)_{0 \leqslant p \leqslant m}$ de diviseurs, une famille $\left(j_{p}\right)_{0 \leqslant p \leqslant m}$ d'éléments de $I$, vérifiant les conditions suivantes:

a) pour tout $p \in\{0, \cdots, m-1\}$, on a $D_{p+1} \equiv D_{p}+C_{i_{p}}$ et

$$
H^{0}\left(C_{j_{p}}, \mathcal{O}_{c_{j p}}\left(-D_{p}\right)\right)=0,
$$

b) on a: $D_{0} \equiv f^{*}(P)$, et $D_{m} \equiv f^{*}(P+n R)-2 L$.

(ii) Il existe un diviseur effectif $E$ sur $S$ passant avec multiplicité 1 par le point $x$ vérifiant: $P \cdot E=R \cdot E=0$ et $E^{2}=-1$. Soit $C_{x}$ la composante irréductible de $E$ passant par $x$. On a: $\mathcal{O}_{C_{x}}(n R-E)=\mathcal{O}_{C_{x}}(\{x\})$.

Il est clair que l'on a l'assertion (i) sauf s'il existe une famille $\left(b_{i}\right)_{i \in I}$ d'entiers $\geqslant 0$ vérifiant:

1) pour tout $i \in I$, on a $b_{i} \leqslant a_{i}$,

2) l'ensemble formé des $i \in I$ tels que $b_{i}<a_{i}$ est non vide. De plus. si $i \in I$ vérifie $b_{i}<a_{i}$, on a:

$$
h^{0}\left(C_{i}, \mathcal{O}_{c_{i}}\left(-f^{*}(P)-\sum_{i \in I} b_{i} C_{i}\right)\right) \geqslant 1 .
$$

Soit $I^{\prime}$ l'ensemble formé des $i \in I$ tels que $b_{i}<a_{i}$. La condition 2) entraîne que pour tout $i \in I^{\prime}$, on a:

$$
C_{i} \cdot\left(f^{*}(P)+\sum_{i \in I} b_{i} C_{i}\right) \leqslant 0 .
$$

Posons $D=\sum_{i \in I} b_{i} C_{i}$. Puisque $f^{*}(P)$ est numeriquement positif, d'après (1), pour tout $i \in I^{\prime}$ on a:

$$
D \cdot C_{i} \leqslant 0 \text {. }
$$

Posons pour chaque $i \in I^{\prime}, e_{i}=a_{i}-b_{i}$; on a: $D+\sum_{i \in I^{\prime}} e_{i} C_{i}=f^{*}(n R)-$ 
$2 L$. D'après la formule $(2)$ on $a: D \cdot\left(\sum_{i \in I^{\prime}} e_{i} C_{i}\right) \leqslant 0$. D'après le $1^{\mathrm{er}}$ pas, on déduit:

$$
D \cdot\left(\sum_{i \in I^{\prime}} e_{i} C_{i}\right)=0 \text {. }
$$

La formule (2) entraîne que pour tout $i \in I^{\prime}$, on a $D \cdot C_{i}=0$. La formule (1) montre que pour tout $i \in I^{\prime}$ on a:

$$
f^{*}(P) \cdot C_{i}=0 \text {. }
$$

La condition 2) entraîne alors que pour tout $i \in I^{\prime}$, on a:

$$
\left.\left.f^{*}(P)\right|_{C_{i}} \equiv D\right|_{C_{i}} \equiv 0 \text {. }
$$

Posons $D^{\prime}=\sum_{i \in I^{\prime}} e_{i} C_{i}$. Soient $M$ et $M^{\prime}$ deux diviseurs effectifs sur $S, a$ et $a^{\prime}$ deux entiers, tels que l'on ait:

$$
\begin{gathered}
D=f^{*}(M)-a L, \quad D^{\prime}=f^{*}\left(M^{\prime}\right)-a^{\prime} L, \quad M+M^{\prime} \equiv n R, \\
\text { et } a+a^{\prime}=2 .
\end{gathered}
$$

$\mathrm{D}^{\prime}$ après la formule (4), on a:

$$
D^{\prime} \cdot f^{*}(P)=M^{\prime} \cdot P=0
$$

La formule (3) montre que:

$$
D \cdot D^{\prime}=M \cdot M^{\prime}-a a^{\prime}=0
$$

Puisque $a+a^{\prime}=2$, on a:

$$
M \cdot M^{\prime} \leqslant 1 \text {. }
$$

Remarquons que $M^{\prime}$ est non nul, car sinon on aurait $a^{\prime}<0$, et la formule (8) serait absurde. La formule (7) et le théorème de l'indice entraînent:

$$
M^{\prime 2} \leqslant-1
$$

La $3^{\text {ème }}$ égalité de (6) montre que:

$$
M \cdot M^{\prime}+M^{\prime 2}=n R \cdot M^{\prime} \geq 0
$$

A l'aide des formules (9) et (10), on déduit:

$$
M \cdot M^{\prime}=1, \quad M^{\prime 2}=-1, \quad R \cdot M^{\prime}=0 .
$$

La formule (8) entraîne:

$$
a=a^{\prime}=1 .
$$

Supposons que $M^{\prime}$ passe au moins deux fois par $\{x\}$. Alors $L$ serait 
composante irréductible de $D^{\prime}$ et d'après la formule (2), on aurait: $D \cdot L$

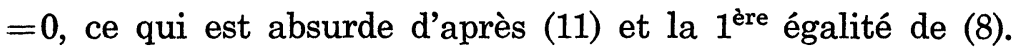

Soient $C_{x}$ la composante de $M^{\prime}$ passant avec multiplicité 1 par $\{x\}$ et $C_{x}^{\prime}$ le transformé strict de $C_{x}$ par $f$. D'après la formule (5), on a:

$$
\left.D\right|_{C_{x}^{\prime}} \equiv 0 \text {, }
$$

d'où l'on déduit: $\mathcal{O}_{C_{x}^{\prime}}\left(f^{*}\left(n R-M^{\prime}\right)\right) \cong \mathcal{O}_{x}^{\prime}(L)$. Cela implique:

$$
\mathcal{O}_{C_{x}}\left(n R-M^{\prime}\right) \cong \mathcal{O}_{C_{x}}(\{x\}) \text {. }
$$

On a donc démontrer le $2^{\text {ème }}$ pas.

$3^{\text {ème }}$ pas. L'assertion (i) du $2^{\text {ème }}$ pas est absurde.

Soit $x \in B(K+P+n R)$. Soient $m$ un entier $>0,\left(D_{p}\right)_{0 \leqslant p \leqslant m}$ une suite de diviseurs sur $S^{\prime},\left(j_{p}\right)_{0 \leqslant p \leqslant m}$ une suite d'éléments de $I$ vérifiant les conditions de l'assertion (i) du $2^{\text {ème }}$ pas. Soit $p \in\{0, \cdots, m-1\}$; on a la suite exacte:

$$
0 \longrightarrow \mathcal{O}_{S^{\prime}}\left(-D_{p+1}\right) \longrightarrow \mathcal{O}_{S^{\prime}}\left(-D_{p}\right) \longrightarrow \mathcal{O}_{c_{j_{p}}}\left(-D_{p}\right) \longrightarrow 0 \text {. }
$$

Montrons par récurrence sur $p$ que $H^{1}\left(S^{\prime}, \mathcal{O}_{S^{\prime}}\left(-D_{p}\right)\right)=0$. D'après le théorème $0-1$, on $\mathrm{a}: H^{1}\left(S^{\prime}, \mathcal{O}_{S^{\prime}}\left(-D_{0}\right)\right)=0$. Supposons que $H^{1}\left(S^{\prime}, \mathcal{O}_{S^{\prime}}\left(-D_{p}\right)\right)$ $=0$. D'après l'assertion (i) a) du $2^{\text {ème }}$ pas, on a: $H^{0}\left(C_{j_{p}}, \mathcal{O}_{c_{j_{p}}}\left(-D_{p}\right)\right)=0$. La suite exacte $(p)$ montre que: $H^{1}\left(S^{\prime}, \mathcal{O}_{S^{\prime}}\left(-D_{p+1}\right)\right)=0$. Cela montre le résultat.

On a: $H^{1}\left(S^{\prime}, \mathcal{O}_{S^{\prime}}\left(-D_{m}\right)\right)=0$. De plus la suite:

$$
0 \longrightarrow \mathcal{O}_{S^{\prime}}\left(f^{*}(K+P+n R)-L\right) \longrightarrow \mathcal{O}_{S^{\prime}}\left(f^{*}(K+P+n R)\right) \longrightarrow \mathcal{O}_{L} \longrightarrow 0
$$

est exacte. Par dualité de Serre, on obtient:

$$
H^{1}\left(S^{\prime}, \mathcal{O}_{S^{\prime}}\left(f^{*}(K+P+n R)-2 L\right)\right) \cong H^{1}\left(S^{\prime}, \mathcal{O}_{S^{\prime}}\left(-D_{m}\right)\right)=0 .
$$

Cela entraîne que l'homomorphisme:

$$
H^{0}\left(S^{\prime}, \mathcal{O}_{S^{\prime}}\left(f^{*}(K+P+n R)\right)\right) \longrightarrow H^{0}\left(L, \mathcal{O}_{L}\right)
$$

est surjectif, ce qui est absurde.

$4^{\text {ème }}$ pas. La démonstration de l'assertion (ii) de la proposition 2-2.

Soit $C$ une courbe fixe du système linéaire $|K+P+n R|$. Alors chaque $x \in C$ appartient à $B(K+P+n R)$. D'après ce qui précéde, il existe un diviseur effectif $E_{x}$ vérifiant $P \cdot E_{x}=R \cdot E_{x}=0$ et $E_{x}=-1$. De plus ce diviseur passe exactement une fois par $x$. Soit $C_{x}$ la composante irréduc- 
tible de $E_{x}$ contenant $x$. D'après le lemme 2-1, il existe un sous-ensemble infini $N$ de $C$, une courbe irréductible $C_{0}$, un diviseur effectif $E$, tels que pour tout $x \in N$, on ait: $E_{x}=E$ et $C_{x}=C_{0}$. Alors, pour tout $x \in N$, on a $x \in C \cap C_{0}$. Cela entraîne que $C=C_{0}$. De plus d'après la formule (12) du $2^{\text {ème }}$ pas, on a:

$$
\mathcal{O}_{C}(\{x\})=\mathcal{O}_{c}(n R-E)
$$

par suite $C$ est une courbe rationnelle lisse vérifiant: $P \cdot C=R \cdot C=0$. Cela termine la démonstration de la proposition 2-2.

3) Sur la résolution minimale de $S$.

Soit $S$ une surface normale et projective vérifiant les hypothèses de la proposition 2-0. Soit $\left(Q_{a}\right)_{a \in A}$ la famille finie des points singuliers de $S, f: S^{\prime} \rightarrow S$ la résolution minimale de ces points singuliers. Pour chaque $a \in A$, notons $\left(E_{i}^{a}\right)_{i \in I_{a}}$ la famille des composantes irréductibles de $f^{-1}\left(Q_{a}\right)$. Notons $K$ le diviseur canonique de $S^{\prime}$. Puisque $f$ est la résolution minimale de $S$, il existe un diviseur effectif $E \in \oplus_{a \in A} \oplus_{i \in I_{a}} N E_{i}^{a}$ tel que: $r K+E \equiv$ $f^{*}(U)$.

Posons $R \equiv f^{*}(D)$ et $P \equiv f^{*}(a D-U)$. Alors $P$ et $R$ sont des diviseurs numériquement positifs, et on a pour tout $a \in A$ et tout $i \in I_{a}$ :

$$
\mathcal{O}_{E_{i}^{a}}(P)=\mathcal{O}_{E_{i}^{a}}(R)=\mathcal{O}_{E_{i}^{a}} .
$$

Comme $S$ est normale, on $a f_{*} \mathcal{O}_{S^{\prime}}=\mathcal{O}_{S}$ et donc, pour tout entier $n$, on a l'isomorphisme:

$$
H^{0}\left(S, \mathcal{O}_{S}(n D)\right) \cong H^{0}\left(S^{\prime}, \mathcal{O}_{S^{\prime}}(n R)\right)
$$

Soient $S_{0}$ le modèle minimal de $S^{\prime}$ et $K_{0}$ l'image inverse du diviseur canonique de $S_{0}$ sur $S^{\prime}$. Reprenons les notations de la proposition 2-1. Il existe une famille de diviseurs effectifs $\left(L_{i}\right)_{i \in I}$ tels que:

$$
K \equiv K_{0}+\sum_{i \in I} L_{i}
$$

Soit $J$ le sous-ensemble formé des $i \in I$ tels que $R \cdot L_{i}=0$. Posons $L=$ $\sum_{i \in J} L_{i}$ et $L^{\prime}=\sum_{i \notin J} L_{i}$. D'après la proposition 2-1, le diviseur $K_{0}+R$ $+L^{\prime}$ est numériquement positif. Posons $E_{0}=E+(r-1) L$.

LEMme 2-2. Pour tout entier $n \geqslant 0$, on $a: B((n+a+r) R) \cap E_{0}=\emptyset$.

Démonstration. Soit $n$ un entier $\geqslant 0$. On a:

$$
(n+a+r) R \equiv K+(r-1)(K+R)+(n+1) R+(a R-r K) .
$$


Par définition, on a:

$$
\begin{aligned}
& K \equiv K_{0}+L+L^{\prime} \\
& a R-r K \equiv f^{*}(a D-U)+E \equiv P+E .
\end{aligned}
$$

En reportant ces égalités dans l'égalité ci-dessus, on obtient:

$$
\begin{aligned}
& (n+a+r) R \\
& \quad \equiv K+(r-1)\left(K_{0}+R+L^{\prime}\right)+(n+1) R+P+E+(r-1) L .
\end{aligned}
$$

Posons $P_{n} \equiv(r-1)\left(K_{0}+R+L^{\prime}\right)+n R+P$; d'après la proposition 2-1, $P_{n}$ est un diviseur numériquement positif. Si $n \geqslant 1$, d'après la remarque 0-1 il vérifie: $P_{n}^{2}>0$. De plus on a:

$$
(n+a+r) R \equiv K+P_{n+1}+E_{0} \text {. }
$$

D'après la définition de $E_{0}$, on a: $\mathcal{O}_{E_{0}}(R) \cong \mathcal{O}_{E_{0}}$. Cela montre que la suite

$$
0 \longrightarrow \mathcal{O}_{S^{\prime}}\left(K+P_{n+1}\right) \longrightarrow \mathcal{O}_{S^{\prime}}((n+a+r) R) \longrightarrow \mathcal{O}_{E_{0}} \longrightarrow 0
$$

est exacte. D'après les remarques précédentes sur $P_{n}$ et le théorème $0-1$, l'homomorphisme:

$$
H^{0}\left(S^{\prime}, \mathcal{O}_{S^{\prime}}((n+a+r) R)\right) \longrightarrow H^{0}\left(E_{0}, \mathcal{O}_{E_{0}}\right)
$$

est surjectif. Comme la fonction constante et égale à 1 appartient à $H^{0}\left(E_{0}, \mathcal{O}_{E_{0}}\right)$, on déduit le résultat annoncé.

Proposition 2-3. Sous les hypothèses de la proposition 2-0, il existe un entier $n_{0}$ tel que pour tout $n \geqslant n_{0}$, le systéme linéaire $|n D|$ est sans point base.

Démonstration. D'après l'isomorphisme (1) de ce paragraphe, il suffit de voir que pour $n$ assez grand, $|n R|$ n'a pas de composantes fixes de dimension 1. Il découle alors des résultats de Zariski [Z] que pour $n$ assez grand, $|n R|$ est sans point base. Posons $P_{0} \equiv(r-1)\left(K_{0}+R+L^{\prime}\right)+P$; si $r \geqslant 2$, on a: $P_{0}^{2}>0$.

Soient $n$ l'entier considéré dans la proposition $2-0$, et $m$ un entier $\geqslant n$. Soit $C$ une courbe fixe du système linéaire $|(m+a+r) R|$. D'après le lemme 2-2, $C$ n'est pas contenue dans $E_{0}$. Remarquons que puisque $S^{\prime}$ est de type général, on a d'après la proposition $0-4: h^{0}\left(S^{\prime}, \mathcal{O}_{S^{\prime}}\left(K+P_{0}+\right.\right.$ $(m+1) R)) \geqslant 1$. D'après la formule $(2)$ de ce paragraphe, on a:

$$
(m+a+r) R \equiv K+P_{0}+(m-n+1) R+n R+E_{0} .
$$


Posons $P_{m} \equiv P_{0}+(m-n+1) R$. D'après ce que l'on vient de voir, $C$ est une composante fixe du système linéaire $\left|K+P_{m}+n R\right|$. Par l'hypothèse c) de la proposition $2-0$, on a: $h^{0}\left(S^{\prime}, \mathcal{O}_{S^{\prime}}(n R)\right) \geqslant 4$. Comme on a $m \geqslant n$, d'après la remarque $0-1, P_{m}$ est un diviseur numériquement positif vérifiant $P_{m}^{2}>0$. D'après la proposition $2-2, C$ est une courbe rationnelle lisse telle que: $P_{m} \cdot C=R \cdot C=0$.

Soient $\left(C_{i}\right)_{i \in I}$ la famille finie des composantes fixes de $|(m+a+r) R|$, $\left(a_{i}\right)_{i \in I}$ la famille des multiplicités des $C_{i}$ dans la partie fixe de $|(m+a+r) R|$, et $M$ sa partie mobile. On a: $(m+a+r) R \equiv M+\sum_{i \in I} a_{i} C_{i}$. Soit $b$ un entier $\in\{2,3\}$; on $a$ :

$$
\begin{aligned}
b(m+a+r) R \equiv & K+P_{0}+(m+1+(b-2)(m+a+r)) R+M \\
& +\sum_{i \in I} a_{i} C_{i}+E_{0} .
\end{aligned}
$$

Posons $G=E_{0}+\sum_{i \in I} a_{i} C_{i}$. Alors puisque chaque $C_{i}$ est rationnelle et lisse, on a: $\mathcal{O}_{G}(R) \cong \mathcal{O}_{G}$. Posons $R_{m} \equiv P_{0}+(m+1+(b-2)(m+a+r)) R$ $+M$. Etant donné que $M$ est numériquement positif, d'après la remarque $0-1, R_{m}$ est numériquement positif et vérifie $R_{m}^{2}>0$. En utilisant le théorème $0-1$, on obtient: $H^{1}\left(S^{\prime}, \mathcal{O}_{S^{\prime}}\left(K+R_{m}\right)\right)=0$. On a la suite exacte:

$$
0 \longrightarrow \mathcal{O}_{S^{\prime}}\left(K+R_{m}\right) \longrightarrow \mathcal{O}_{S^{\prime}}(b(m+a+r) R) \longrightarrow \mathcal{O}_{G} \longrightarrow 0 \text {. }
$$

L'homomorphisme: $H^{0}\left(S^{\prime}, \mathcal{O}_{S^{\prime}}(b(m+a+r) R)\right) \rightarrow H^{0}\left(G, \mathcal{O}_{G}\right)$ est surjectif. Comme la fonction constante et égale à 1 appartient à $H^{\circ}\left(G, \mathcal{O}_{G}\right)$, on déduit:

$$
B(b(m+a+r) R) \cap G=\emptyset .
$$

Mais on a: $B(b(m+a+r) R) \subset B((m+a+r) R)$. Cela entraîne:

$$
\operatorname{dim} B(b(m+a+r) R)=0 .
$$

Puisque pour tout $s \geqslant 7(n+a+r)$ il existe deux entiers $p$ et $q \geqslant n$ vérifiant $s=2(p+a+r)+3(q+a+r)$, on obtient le résultat annoncé.

4) La démonstration de la proposition 2-0

Remarque 2-1. Soit $C$ une courbe sur $S^{\prime}$ telle que $R \cdot C=0$. Alors on a:

$$
\mathcal{O}_{C}(R) \cong \mathcal{O}_{C}
$$

En effet, d'après la proposition 2-3, il existe un entier $m_{0}$ tel que pour $m \geqslant m_{0},|m R|$ n'ait pas de point base. Soit $m$ un entier $\geqslant m_{0}$; on a:

$$
\mathcal{O}_{c}((m+1) R) \cong \mathcal{O}_{c}(m R) \cong \mathcal{O}_{C},
$$


ce qui montre le résultat.

LEMme 2-3. Soit $E$ un diviseur effectif vérifiant $R \cdot E=0$ et $E^{2}=-1$. Alors $E$ est 1-connexe au sens de [Ra].

Démonstration. Soient $D_{1}$ et $D_{2}$ deux diviseurs effectifs non nuls, tels que: $E=D_{1}+D_{2}$. Puisque $R$ est numériquement positif, on a $R \cdot D_{1}=$ $R \cdot D_{2}=0$. Comme $R^{2}>0$, le théorème de l'indice implique: $D_{1}^{2} \leqslant-1$ et $D_{2}^{2} \leqslant-1$. L'égalité: $-1=E^{2}=D_{1}^{2}+2 D_{1} \cdot D_{2}+D_{2}^{2}$ montre que: $D_{1} \cdot D_{2} \geqslant 1$, ce qui termine la démonstration.

Proposition 2-4. Sous l'hypothèse de la proposition 2-0, le système linéaire $|(m+a+r) D|$ est sans point base dans les deux cas suivants:

(i) $r \geqslant 2$ et $m \geqslant n-1$,

(ii) $r=1$ et $m \geqslant n$.

Démonstration. D'après l'isomorphisme (1) du paragraphe 3 de ce chapitre, il suffit de montrer que $|(m+a+r) R|$ n'a pas de point base. Soient $m$ un entier $\geqslant n-1$ si $r \geqslant 2$ ou $\geqslant n$ si $r=1$, et $x$ un point fermé de $B((m+a+r) R)$. Par le lemme 2-2, on a $x \notin E_{0}$. Comme précédemment posons $P_{0} \equiv(r-1)\left(K_{0}+R+L^{\prime}\right)+P$ et $P_{m} \equiv P_{0}+(m-n+1) R$. D'après la formule (2) du paragraphe 3 de ce chapitre, on a:

$$
\begin{aligned}
(m+a+r) R & \equiv K+P_{0}+(m-n+1) R+n R+E_{0} \\
& \equiv K+P_{m}+n R+E_{0} .
\end{aligned}
$$

D'après la remarque $0-1$ et les conditions (i) ou (ii), $P_{m}$ est un diviseur numériquement positif vérifiant $P_{m}^{2}>0$. De plus la proposition 0-4 implique: $\left|K+P_{m}+n R\right| \neq \emptyset$. On en déduit que $X$ est point base du système linéaire $\left|K+P_{m}+n R\right|$. D'après l'hypothèse $c$ ) de la proposition 2-0, on a: $h^{0}\left(S^{\prime}, \mathcal{O}_{S^{\prime}}(n R)\right) \geqslant 4$. La proposition 2-1 implique qu'il existe un diviseur effectif $E$ vérifiant: $R \cdot E=P_{m} \cdot E=0$ et $E^{2}=-1$. De plus $E$ passe par le point $x$. La remarque 2-1 montre que:

$$
\mathcal{O}_{E}(R)=\mathcal{O}_{E}
$$

Montrons que $E \cap E_{0}=\emptyset:$ D'après le lemme 2-3, $E$ est connexe; comme toutes les sections de $H^{0}\left(S^{\prime}, \mathcal{O}_{S^{\prime}}((m+a+r) R)\right)$ s'annulent au point $x$, d'après la formule (1), ces sections s'annulent aussi le long de $E$. D'après le lemme 2-2, on a $E \cap E_{0}=\emptyset$; on obtient: $(m+a+r) R \cdot E=K \cdot E+P_{m} \cdot E$ $+n R \cdot E+E_{0} \cdot E=K \cdot E=0$. Cela implique que l'on a: $E^{2} \equiv 0(\bmod 2)$. 
Comme $E^{2}=-1$, on obtient une contradiction, ce qui démontre la proposition 2-4.

Il est clair que la proposition 2-4 entraîne la proposition 2-0 par la formule (1) du début de la troisième section de ce chapitre. Cela termine la démonstration de la proposition 2-0.

\section{La démonstration des théorèmes 1 et 2}

1) La démonstration du théorème 1:

Reprenons les notations données en (0-1) au chapitre 0.

Proposition 3-1. Soit $m$ un entier $\geqslant 17$; il existe une surface irréductible, normale, $\mathbf{Q}$-Gorenstein de type général au sens des définitions 2-1 et 2-2 dans le système linéaire $|m H|$ n'ayant que des singularités isolées.

Démonstration. D'après la proposition $0-3$ (i), on a $|4 H| \neq \emptyset$ et $|5 H|$ $\neq \emptyset$. D'après l'assertion (ii) de la proposition $0-3$, le système linéaire $|m H|$ n'est pas composé d'un pinceau, et on a: $r H-H \equiv(r-1) H$. Les hypothèses de la proposition 1 sont donc satisfaites en y faisant $D=H$. Soit $S \in|m H|$ une surface irréductible n'ayant que des singularités isolées. Puisque $X$ est de Cohen-Macaulay, $S$ l'est aussi. Comme $S$ n'a que des singularités isolées $S$ est normale. Soit $f: Y \rightarrow X$ la résolution des singularités de $X$ vérifiant les hypothèses données en (0-1). Soient $Y^{\prime}$ un modèle birationnel de $Y, f^{\prime}: Y^{\prime} \rightarrow Y$ un morphisme birationnel composé d'une suite finie d'éclatements de centres lisses, vérifiant les conditions suivantes: Posons $p=f \circ f^{\prime}$; alors le transformé strict $S^{\prime}$ de $S$ par $p$ est lisse.

Soit $K_{Y^{\prime}}$ le diviseur canonique de $Y^{\prime}$; par hypothèse, il existe un diviseur effectif $E$ tel que: $r K_{Y^{\prime}} \equiv p^{*}(H)+E$. Soit $K^{\prime}$ le diviseur canonique de $S^{\prime}$ et notons $G=\left.\left(r S^{\prime}+E\right)\right|_{S^{\prime}}$. La formule d'adjonction donne:

$$
r K^{\prime} \equiv G+\left.p^{*}(H)\right|_{S^{\prime}} \text {. }
$$

Comme $G$ est un diviseur effectif, cela implique que $S^{\prime}$ est de type général. Soit $\omega_{S}$ le faisceau dualisant de $S$; on a: $\omega_{S}^{\otimes r}=\omega_{X}^{\otimes r}(r S) \otimes \mathcal{O}_{S}$. Soient $\mathscr{L}$ le bidual de $\omega_{S}^{\otimes r}$ et $A$ la réunion du lieu singulier de $S$ et des points singuliers de $X$ d'indice $>1$.

Alors $\operatorname{dim}(A)=0$ et si l'on pose $\mathscr{U}=X-A$, on a: $\left.\mathcal{O}_{\mathscr{U}}(H) \cong \omega_{X}^{\otimes r}\right|_{\mathscr{U}}$. Posons aussi $\mathscr{V}=S \cap \mathscr{U}$; on déduit:

$$
\left.\left.\left.\mathscr{L}\right|_{r} \cong \omega_{S}^{\otimes r}\right|_{r} \cong \mathcal{O}_{\mathscr{U}}(H+r S)\right|_{r} \text {. }
$$


Puisque la codimension de $\mathscr{V}$ dans $S$ est $\geqslant 2$, on a: $\mathscr{L} \cong \mathcal{O}_{X}(H+r S) \otimes \mathcal{O}_{S}$. Par suite $\mathscr{L} \cong \mathcal{O}_{S}((1+r m) H)$ et $S$ est $Q$-Gorenstein. Cela achève de montrer la proposition $3-1$.

LEMмE 3-1. Soient $m$ un entier $\geqslant 17, S$ une surface irréductible dans $|m H|$. Alors pour tout entier $p$ vérifiant: $4 \leqslant p<m$, on $a: h^{0}\left(S, \mathcal{O}_{S}(m H)\right)$ $\geqslant 7$.

Démonstration. Soient $S, p$ et $m$ vérifiant les hypothèses du lemme. On a la suite exacte:

$$
0 \longrightarrow \mathcal{O}_{X}((p-m) H) \longrightarrow \mathcal{O}_{X}(p H) \longrightarrow \mathcal{O}_{S}(p H) \longrightarrow 0
$$

D'après la proposition $0-2$, on a l'isomorphisme:

$$
H^{0}\left(X, \mathcal{O}_{X}(p H)\right) \stackrel{\sim}{\longrightarrow} H^{0}\left(S, \mathcal{O}_{S}(p H)\right) .
$$

La proposition 0-3 implique: $h^{0}\left(S, \mathcal{O}_{S}(p H)\right) \geqslant 7$, ce qui démontre le résultat.

Proposition 3-2. Si $r \geqslant 2$, pour tout entier $m \geqslant 34 r+3$, le système linéaire $|\mathrm{mH}|$ est sans point base.

Démonstration. Soit $m$ un entier $\geqslant 34 r+3$. Il existe un entier $s \geqslant r$ et un entier $p$ vérifiant $17 \leqslant p \leqslant 33$, tels que l'on ait: $m=17 s+p$. Soit $Q$ un point base du système linéaire $|m H|$. Comme on a: $s|17 H|+|p H|$ $\subset|m H|$, il existe un entier $b \in\{17, p\}$ tel que $Q$ soit point base de $|b H|$.

D'après la proposition $3-1$, il existe une surface $S$ normale $Q$-Gorenstein de type général dans $|b H|$. Posons $D=\left.H\right|_{s}$. D'après la démonstration de la proposition 3-1, le bidual de $\omega_{S}^{\otimes r}$ est isomorphe à: $\mathcal{O}_{S}((1+b r) D)$. Posons $a=1+b r$ et $U=a D$; alors on a: $a D-U \equiv 0$, et $D^{2}=b H^{3}>0$. De plus $D$ est numériquement positif; d'après le lemme $3-1$, on a pour tout entier $n$ verifiant $4 \leqslant n<b: h^{0}\left(S, \mathcal{O}_{S}(n D)\right) \geqslant 7$. La proposition 2-0 implique que pour tout entier $q \geqslant a+r+3$, le faisceau $\mathcal{O}_{S}(q D)$ est engendré par ses sections globales. On a la suite exacte:

$$
0 \longrightarrow \mathcal{O}_{X}((m-b) H) \longrightarrow \mathcal{O}_{X}(m H) \longrightarrow \mathcal{O}_{S}(m D) \longrightarrow 0 \text {. }
$$

D'après la proposition 0-2, l'homomorphisme: $H^{0}\left(X, \mathcal{O}_{X}(m H)\right) \rightarrow H^{0}\left(S, \mathcal{O}_{S}(m D)\right)$ est surjectif. Montrons que $m \geqslant a+r+3$.

1) Supposons que $b=17$.

Alors $a+r+3=17 r+r+3=18 r+3$, et il est clair que: $m \geqslant$ $a+r+3$. 
2) Supposons que $b=p$.

Alors on a: $a+r+3=p r+r+3=(p+1) r+3$. Mais $p+1 \leqslant 34$, par suite, puisque par hypothèse $m \geqslant 34 r+3$, on a: $m \geqslant a+r+3$, ce qui démontre le résultat.

Cela donne une contradiction, et démontre la proposition 3-2. Le théorème 1 est donc démontré dans le cas où $r \geqslant 2$. Le théorème 2 couvre le cas $r=1$.

2) La démonstration $d u$ théorème 2.

Nous supposerons désormais $r=1$. Alors $\omega_{X}$ est un faisceau inversible et est isomorphe à $\mathcal{O}_{X}(H)$. Soit $m$ un entier $\geqslant 17$; d'après la proposition $3-1$, il existe une surface $S$ irréductible, normale, de Gorenstein dans le système linéaire $|m H|$. Posons $R=\left.H\right|_{S}$, alors si l'on note $\omega_{S}$ le faisceau dualisant de $S$, on a: $\omega_{S}=\mathcal{O}_{S}((m+1) R)$. Par hypothèse $R$ est ample. De plus le lemme $3-1$ montre que pour $4 \leqslant p<m$, on a: $h^{0}\left(S, \mathcal{O}_{s}(p R)\right) \geqslant 5$. Les hypothèses de la proposition $2-0$ sont réalisées pour $S$. Distinguons les deux cas suivants:

$1^{\text {er }}$ cas. Supposons que le système linéaire $|2 m H|$ ait un point base $Q$. Alors $Q$ est aussi point base $\operatorname{de}|m H|$ et $Q \in S$. On a la suite exacte:

$$
0 \longrightarrow \mathcal{O}_{X}(m H) \longrightarrow \mathcal{O}_{X}(H+S+(m-1) H) \longrightarrow \omega_{S}((m-1) R) \longrightarrow 0 .
$$

D'après les considérations du début et le théorème $0-1$, on obtient une surjection:

$$
H^{0}\left(X, \mathcal{O}_{X}(H+S+(m-1) H)\right) \longrightarrow H^{0}\left(S, \mathcal{O}_{S}((m-1) R) .\right.
$$

D'après la proposition $2-0$, le faisceau $\omega_{S}((m-1) R)$ est engendré par ses sections globales. Mais on a: $H+S+(m-1) H \equiv 2 m H$, ce qui donne une contradiction.

$2^{\text {ème }}$ cas. Supposons que le système linéaire $|(2 m+1) H|$ ait un point base $Q$. Comme $|(m+1) H|+|m H| \subset|(2 m+1) H|$ soit $Q$ est point base de $|m H|$, soit il est point base de $|(m+1) H|$.

1) Si $Q$ est point base de $|m H|, Q \in S$ et on a la suite exacte:

$$
0 \longrightarrow \mathcal{O}_{X}(H+m H) \longrightarrow \mathcal{O}_{X}(H+S+m H) \longrightarrow \omega_{S}(m R) \longrightarrow 0
$$

On conclut alors comme précédemment.

2) Si $Q$ est point base de $|(m+1) H|$, on remplace $m$ par $(m+1)$ et on peut alors supposer que $Q \in S$. On a la suite exacte: 


$$
\begin{aligned}
0 \longrightarrow \mathcal{O}_{X}(H+(m-2) H) \longrightarrow \mathcal{O}_{X}(H+S+(m-2) H) \\
\\
\longrightarrow \omega_{S}((m-2) R) \longrightarrow 0 .
\end{aligned}
$$

On conclut alors comme pour le cas précédent.

Cela termine la démonstration du théorème 2 .

\section{REFERENCES}

[B-1] X. Benveniste, Sur les variétés de dimension 3 de type général dont le diviseur canonique est numériquement positif, Math. Ann., 266 (1984), 479-497.

[B-2] - Sur l'anneau canonique de certaines variétés de dimension 3, Invent. Math., 73 (1983), 153-164.

[B-3] — Sur la décomposition de Zariski en dimension 3, A paraître.

[E] R. Elkik, Rationnalité des singularités canoniques, Invent. Math., 47 (1978), 139-147.

[F] T. Fujita, Semi-positive line bundles, A paraître.

[Ha] R. Hartshorne, Algebraic Geometry, G.T.M. 52, Springer Verlag (1977).

[Hi] H. Hironaka, Resolution of an algebraic variety over a field of characteristic zero (I, II), Ann. of Math., 79 no. 1 (1964).

[K] Y. Kawamata, A generalisation of Kodaira Ramanujam's vanishing theorem, Math. Ann., 261 (1983), 43-46.

[K-1] — The cone of curves of algebraic varieties, Ann. of Math., 119 (1984), 603633.

[M] D. Mumford, The topology of normal singularities of an algebraic surface and a criterion for simplicity, I.H.E.S. Publ. Math. no. 9 (1971).

[Ra] C. P. Ramanujam, Supplément à l'article "Remarks on Kodaira Vanishing Theorem", J. Indian, Math. Soc., 38 (1974), 121-124.

[R-1] M. Reid, Canonical 3-folds, Proceedings de "Journees de Géometrie Algébrique" Angers 1979, A. Beauville éditeur, 273-310, Sijthoff and Noordhoff 1980.

[R-2] - Minimal models of canonical 3-folds, Adv. Stu. in Pure Math., Algebraic Varieties and Analytic Varieties, ed. S. Iitaka, 1 (1983), 131-180.

[S] N. Shepherd-Barron, Canonical 3-fold singularities are Cohen-Macaulay, Warwick, preprint.

[V] E. Viehweg, Vanishing theorem, J. Reine Angew. Math., 335 (1982).

[Z] O. Zariski, The Theorem of Riemann-Roch for high multiples of an effective divisor on an algebraic surface, Ann. of Math., 76 (1962), 560-615.

Centre de Mathématiques

Ecole Polytechnique

F-91 128 PALAISEAU

France 